ENSL-00325444

\title{
Supergravities without an Action: Gauging the Trombone
}

\author{
Arnaud Le Diffon and Henning Samtleben \\ Université de Lyon, Laboratoire de Physique, ENS Lyon, \\ 46 allée d'Italie, F-69364 Lyon CEDEX 07, France \\ arnaud.le_diffon, henning.samtleben @ens-lyon.fr
}

\begin{abstract}
We present a systematic account of supergravity theories in which the global scaling symmetry is gauged. This generalizes the standard gaugings of nonabelian off-shell symmetries. A particular feature of these theories is an additional positive contribution to the effective cosmological constant.

As the scaling symmetry is an on-shell symmetry, the resulting gaugings do no longer possess an action. We develop the algebraic framework for the maximal theories in various dimensions and construct explicit solutions to the algebraic consistency constraints — related to "pure-spinor-like" structures for the exceptional groups. As an example, we explicitly work out the modified supersymmetry transformation rules and equations of motion in three dimensions. Finally, we speculate about the role of these theories from the perspective of very extended Kac-Moody algebras.
\end{abstract}




\section{Contents}

1 Introduction $\quad 1$

2 Gauging the scaling symmetry 5

2.1 Gravity sector . . . . . . . . . . . . . . . 5

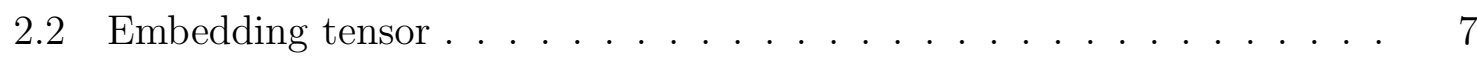

2.3 Quadratic constraints . . . . . . . . . . . . . 9

3 Algebraic structure in various dimensions $\quad \mathbf{1 0}$

$3.1 \quad D=6 \ldots \ldots \ldots \ldots \ldots \ldots \ldots$

$3.2 D=5 \ldots \ldots \ldots \ldots \ldots \ldots \ldots$

$3.3 D=4 \ldots \ldots \ldots \ldots \ldots \ldots \ldots$

$3.4 \quad D=3 \ldots \ldots \ldots \ldots \ldots \ldots \ldots \ldots \ldots$

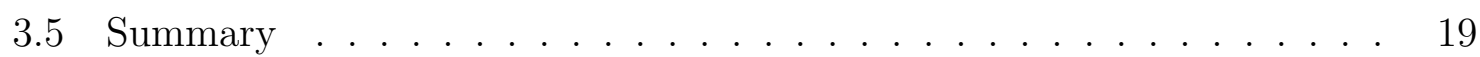

4 Supersymmetry $\quad 20$

4.1 The three-dimensional theory ................. . . . . . . . . . . . . . . .

4.2 Implications of the quadratic constraint . . . . . . . . . . . . . . . . . 22

4.3 Supersymmetry algebra. . . . . . . . . . . . . . . . . . . . . . . . . . . . . . . . . .

4.4 Equations of motion . . . . . . . . . . . . . . 26 26

5 Conclusions and Outlook $\quad 29$

A Algebra conventions

A.1 $\mathrm{E}_{8(8)}$ conventions. . . . . . . . . . . . . . . . . . . . . . . . . . . . . . . . . .

A.2 $\mathrm{E}_{7(7)}$ conventions and identities. . . . . . . . . . . . . . . . . . . . . . . . . . . .

A.3 $\mathrm{E}_{8(8)}$ algebra in the $\mathrm{E}_{7(7)} \times \mathrm{SL}(2)$ basis $\ldots . . . . . . . . . . . .33$

B Solution of the $\mathrm{E}_{8(8)}$ constraint (3.53) 34

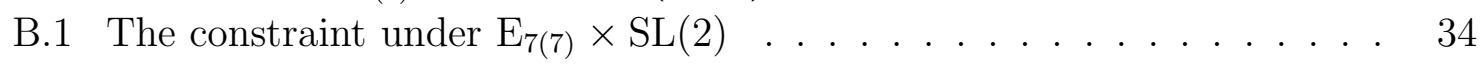

B.2 Solving the constraint . . . . . . . . . . . . . 36

\section{Introduction}

Einstein's equations of general relativity possess a well-known global symmetry under conformal rescaling of the metric

$$
g_{\mu \nu} \rightarrow \Lambda^{2} g_{\mu \nu}
$$

with constant $\Lambda$. As the Einstein-Hilbert Lagrangian scales according to $\mathcal{L}_{\mathrm{EH}} \rightarrow$ $\Lambda^{D-2} \mathcal{L}_{\mathrm{EH}}$, this symmetry is off-shell realized only in $D=2$ space-time dimensions. 
The global scaling symmetry extends to supergravity theories in all dimensions, with gravitinos and matter fields scaling with different weights under (1.1), in particular, $p$-forms scale with weight $p$ and scalars fields are invariant. In this context it is often referred to as a trombone symmetry and plays an important role e.g. among the spectrum-generating symmetries for the fundamental BPS solutions [1].

In addition, the maximal supergravity theories admit rather large global symmetry groups, given by the exceptional groups $\mathrm{G}=\mathrm{E}_{n(n)}$, which (at least in odd dimensions) are genuine off-shell symmetries of the action 1 These have first been revealed in fourdimensional maximal supergravity [2] and we will refer to them as duality (or CremmerJulia) symmetries, see [3] for a review. In fact, the trombone symmetry (1.1) plays an important role in the realization of the Cremmer-Julia groups $\mathrm{E}_{n(n)}$ in the various dimensions. Recall that maximal supergravities are obtained by dimensional reduction of the eleven-dimensional theory [4] on an $n$-torus. From the eleven-dimensional point of view there are two scaling symmetries inherited to the $D=(11-n)$-dimensional theory. Apart from the trombone symmetry (1.1) of eleven-dimensional supergravity, a rescaling of the $n$-torus

$$
y^{i} \rightarrow \alpha y^{i}, \quad i=1, \ldots, n, \quad \text { for the coordinates } y^{i} \text { of the torus }
$$

is part of the eleven-dimensional diffeomorphisms and translates into a particular rescaling of the $D$-dimensional fields. From the $D$-dimensional point of view, it is however more natural to consider particular combinations of the two scaling symmetries (1.1), (1.2): Choosing $\alpha=\Lambda$ defines a scaling which leaves the scalars of the $D$-dimensional theory invariant - this is the $D$-dimensional trombone symmetry (1.1). On the other hand, choosing $\alpha=\Lambda^{9 / n}$ defines a scaling which leaves the $D$-dimensional metric (in the Einstein frame) invariant 2 this symmetry is part of the Cremmer-Julia group embedded as $\mathrm{GL}(1) \subset \mathrm{GL}(n) \subset \mathrm{E}_{n(n)}$. This shows how higher-dimensional trombone symmetries naturally merge with the lower-dimensional duality groups.

It is well known that certain subgroups of the global Cremmer-Julia symmetry groups $\mathrm{E}_{n(n)}$ may be promoted to local symmetries while preserving all supersymmetries [5, 6, 7]. The resulting gauged supergravities exhibit non-abelian gauge groups, additional couplings and in particular a scalar potential. The construction of these theories can be systematically performed using the group-theoretical framework of [8, 9, 10, 11] which allows to characterize the various gaugings in terms of a single tensorial object, the embedding tensor $\Theta$, subject to a number of algebraic constraints that encode the consistency of the theory. In view of the close relation of the Cremmer-Julia groups $\mathrm{E}_{n(n)}$ and the trombone symmetry (1.1) in dimensional reduction, it seems natural to also consider the possible gaugings of the trombone symmetry. This is what we are going to address in this paper.

\footnotetext{
${ }^{1}$ The notation $\mathrm{E}_{n(n)}$ refers to the split form of the exceptional group $\mathrm{E}_{n}$.

${ }^{2}$ In the reduction to $D=2$ dimensions $(n=9)$, this scaling degenerates as a sign of the fact that in two dimensions the theory cannot be cast into the Einstein frame.
} 


\begin{tabular}{c||c|c|c||c|c|}
$D$ & $\mathrm{G}$ & $\mathcal{R}_{\text {adj }}$ & $\mathcal{R}_{\mathrm{v}}$ & $\Theta$ & $\theta$ \\
\hline 7 & $\mathrm{SL}(5)$ & $\mathbf{2 4}$ & $\mathbf{1 0}^{\prime}$ & $\mathbf{1 5}+\mathbf{4 0}^{\prime}$ & $\mathbf{1 0}$ \\
6 & $\mathrm{SO}(5,5)$ & $\mathbf{4 5}$ & $\mathbf{1 6}_{c}$ & $\mathbf{1 4 4}_{c}$ & $\mathbf{1 6}_{s}$ \\
5 & $\mathrm{E}_{6(6)}$ & $\mathbf{7 8}$ & $\mathbf{2 7}^{\prime}$ & $\mathbf{3 5 1}^{\prime}$ & $\mathbf{2 7}$ \\
4 & $\mathrm{E}_{7(7)}$ & $\mathbf{1 3 3}$ & $\mathbf{5 6}$ & $\mathbf{9 1 2}$ & $\mathbf{5 6}$ \\
3 & $\mathrm{E}_{8(8)}$ & $\mathbf{2 4 8}$ & $\mathbf{2 4 8}$ & $\mathbf{1 + 3 8 7 5}$ & $\mathbf{2 4 8}$ \\
\cline { 5 - 6 } 2 & $\mathrm{E}_{9(9)}$ & $\mathcal{R}_{\text {adj }}$ & $\boldsymbol{\Lambda}_{1}$ & \multicolumn{2}{|c}{$\boldsymbol{\Lambda}_{1 *}$}
\end{tabular}

Table 1: The embedding tensor in maximal supergravity in various dimensions. $\mathcal{R}_{\text {adj }}$ and $\mathcal{R}_{\mathrm{v}}$ denote the adjoint representation of the global symmetry group $\mathrm{G}$ and the representation in which the vector fields transform, respectively. The tensors $\Theta$ and $\theta$ denote the components of the embedding tensor, the latter also induce a gauging of the trombone symmetry (1.1).

Certain theories with local trombone symmetry have already appeared in the literature. A straightforward way to obtain such theories is by performing a standard Scherk-Schwarz reduction [12] twisting the fields with the higher-dimensional on-shell symmetry (1.1). Applying this to the circle reduction of eleven-dimensional supergravity gives rise to a one-parameter deformation of the ten-dimensional IIA theory with maximal supersymmetry [13, 14] which is different from Romans' massive supergravity [15]. In particular, this theory does not have an action and admits a de Sitter vacuum. According to the discussion above, from the ten-dimensional point of view this theory corresponds to the gauging of a linear combination of the $D=10$ trombone symmetry and the off-shell GL(1) symmetry. It has been further studied in [16, 17, 18, 19]. Other examples of such theories have been obtained in lower dimensions by studying analogous generalized Scherk-Schwarz reductions to nine and to six dimensions [20, 21, 22].

In this paper we will set up a systematic framework for the classification and construction of these theories. We follow the group-theoretical approach of [8, 9, 10, 11], in which theories with a local trombone symmetry (1.1) simply correspond to the introduction of additional components $\theta_{M}$ in the embedding tensor. This allows to straightforwardly derive consistency conditions on such gaugings and to construct explicit examples exploiting the structure of the underlying symmetry groups. As it turns out, the additional components in the embedding tensor generically induce a simultaneous gauging of the trombone symmetry (1.1) and a subgroup of the duality group G.

In table 1 we have collected the representations in which the embedding tensor transforms for the maximal supergravities in various dimensions. The standard gaugings are described by a tensor $\Theta$ inducing gauge groups that are subgroups of the duality group $\mathrm{G}$ and do not include the trombone symmetry (1.1). This tensor transforms in a particular subrepresentation of the tensor product $\mathcal{R}_{\text {adj }} \otimes \mathcal{R}_{\mathrm{v} *}$ of the adjoint representation of $\mathrm{G}$ and the representation dual to the vector fields of the theory. The 
corresponding theories have been constructed in [8, 10, 23, 24, 25, 26]. The theories we will construct in this paper allow for additional components in the embedding tensor, combining into a vector $\theta$ which transforms in the representation $\mathcal{R}_{\mathrm{v} *}$. Note that in two dimensions the two objects $\Theta$ and $\theta$ coincide (in fact, this observation triggered the present investigation). As a consequence, in two dimensions gaugings generically include a local trombone symmetry (1.1), in accordance with the fact that in $D=2$ dimensions this symmetry becomes off-shell - more precisely it builds the central extension of the affine global symmetry group $\mathrm{E}_{9(9)}$ [27]. Moreover, this indicates that the new theories we present in this paper are particularly interesting from the unifying point of view of the extended Kac-Moody algebras $\mathrm{E}_{10}$ [28] and $\mathrm{E}_{11}$ [29], conjectured to underlie eleven-dimensional supergravity and its compactifications. We will come back to this in the conclusions.

The rest of this paper is organized as follows. In section 2 we set up the general formalism in order to describe a theory with local scaling symmetry (1.1). In the gravity sector this introduces new minimal couplings between the metric and a vector field which modify the Einstein equations. In the full theory we describe the simultaneous gauging of a subgroup of the duality group $G$ and the scaling symmetry (1.1) by an embedding tensor $\widehat{\Theta}$ which completely encodes the theory. With respect to the standard constructions, the possibility of a local scaling symmetry translates into a set of additional components $\theta_{M}$ of the embedding tensor. We derive the quadratic constraints on the embedding tensor which encode consistency of the gaugings.

In section 3 we work out the details of the construction for all maximal supergravities in dimensions $6 \geq D \geq 3$. The analysis relies on the particular properties of the global symmetry groups $\mathrm{SO}(5,5), \mathrm{E}_{6(6)}, \mathrm{E}_{7(7)}$, and $\mathrm{E}_{8(8)}$ of these theories. In particular, we investigate the class of gaugings which is exclusively triggered by the new components $\theta_{M}$ of the embedding tensor. In this case, the quadratic consistency constraints reduce to a simple set of equations that for $\mathrm{SO}(5,5)$ reduce to the well-known "purespinor" condition and to its higher-rank analogues in the other dimensions. We present the explicit solution of these quadratic constraints.

Subsequently, in section 4 we analyze the compatibility of the gaugings with supersymmetry. For the case of the three-dimensional theory we derive the full set of deformed equations of motion and show closure of the supersymmetry algebra. We find that a particular effect of the theories with local scaling symmetry is a positive contribution to the effective cosmological constant. We close the paper with some speculations on the possible role of these new theories in the unifying framework of the extended Kac-Moody algebras $\mathrm{E}_{10}$ and $\mathrm{E}_{11}$. 


\section{Gauging the scaling symmetry}

In supergravity theories, the trombone symmetry (1.1) extends to the full bosonic field content: the metric and the antisymmetric $p$-forms infinitesimally scale as

$$
\delta g_{\mu \nu}=2 \lambda g_{\mu \nu}, \quad \delta A_{\mu_{1} \ldots \mu_{p}}=p \lambda A_{\mu_{1} \ldots \mu_{p}}
$$

respectively, with a constant parameter $\lambda$, while scalar fields remain invariant. The fermionic fields on the other hand transform as

$$
\delta \psi_{\mu}=\frac{1}{2} \lambda \psi_{\mu}, \quad \delta \chi=-\frac{1}{2} \lambda \chi,
$$

for gravitinos $\psi_{\mu}$ and spin-1/2 fermions $\chi$, respectively. It is easy to check that under this symmetry all kinetic terms of the Lagrangian scale homogeneously as

$$
\delta \mathcal{L}_{\text {kin }}=(D-2) \lambda \mathcal{L}_{\text {kin }} .
$$

It is a non-trivial property of supergravity theories that also all interaction terms scale with the same weight. In particular, this restricts the topological terms to twoderivative terms.

In the following we will consider gaugings of supergravity in which the trombone symmetry (2.1), (2.2) becomes a local symmetry. Following the standard procedure, this is achieved by introducing covariant derivatives

$$
D_{\mu} \equiv \partial_{\mu}-\mathcal{A}_{\mu} t_{0}
$$

where $t_{0}$ denotes the generator of the trombone symmetry. However, this cannot be the full answer. Consistency implies that the vector field $\mathcal{A}_{\mu}$ itself must not be charged under the symmetry it is gauging. This shows already that the local gauge symmetry cannot simply be the scaling symmetry (2.1), (2.2) under which all vector fields are charged. Rather, gauging of the scaling symmetry must be accompanied by a gauging of other generators of the global symmetry group $\mathrm{G}$ of the theory - which however is invisible in the gravity sector. Indeed, this is what we will find in the following.

\subsection{Gravity sector}

To begin with, we will study the gravity sector with local scaling symmetry (2.1), i.e. introduce covariant derivatives (2.4) in Einstein's equations. Since eventually we are interested in supergravity, we use the formulation in terms of the vielbein $e_{\mu}{ }^{a}$ with curved indices $\mu, \nu, \ldots$ and flat indices $a, b, \ldots$ As a first step, the standard spin connection $\omega_{\mu}^{a b}$ defined by

$$
0 \stackrel{!}{\equiv} \nabla(\omega)_{[\mu} e_{\nu]}^{a} \equiv \partial_{[\mu} e_{\nu]}^{a}+\omega_{[\mu}^{a b} e_{\nu] b},
$$

is replaced by a covariantized object $\widehat{\omega}_{\mu}^{a b}$ defined by

$$
0 \stackrel{!}{\equiv} D_{[\mu} e_{\nu]}^{a}+\widehat{\omega}_{[\mu}^{a b} e_{\nu] b}
$$


with the covariant derivative $D_{\mu}$ from (2.4). Explicitly, this yields

$$
\widehat{\omega}_{\mu}^{a b}=\omega_{\mu}^{a b}-2 e_{\mu}^{[a} e^{b] \nu} \mathcal{A}_{\nu},
$$

for the modified spin connection which is uncharged under the scaling symmetry. It is important to note that this covariantization of the spin connection may equivalently be interpreted as the adding of a torsion trace term $T_{\mu \nu}^{a}=2 \mathcal{A}_{[\mu} e_{\nu]}^{a} 3$ Likewise, we define the covariantized Riemann tensor as

$$
\begin{aligned}
\widehat{\mathcal{R}}_{\mu \nu}{ }^{a b} & \equiv 2 \partial_{[\mu} \widehat{\omega}_{\nu]}^{a b}+2 \widehat{\omega}_{[\mu}{ }^{a c} \widehat{\omega}_{\nu] c}{ }^{b} \\
& =R_{\mu \nu}{ }^{a b}+4 e_{[\mu}{ }^{[a} \nabla(\omega)_{\nu]} \mathcal{A}^{b]}+4 e_{[\mu}{ }^{[a} \mathcal{A}_{\nu]} \mathcal{A}^{b]}-2 e_{[\mu}{ }^{a} e_{\nu]}{ }^{b} \mathcal{A}_{\lambda} \mathcal{A}^{\lambda} .
\end{aligned}
$$

By construction it is invariant under gauge transformations

$$
\delta e_{\mu}{ }^{a}=\lambda(x) e_{\mu}{ }^{a}, \quad \delta \mathcal{A}_{\mu}=\partial_{\mu} \lambda(x) .
$$

The covariantized Riemann tensor (2.8) no longer possesses the symmetries of the standard Riemann tensor: the first Bianchi identity is modified to

$$
\widehat{\mathcal{R}}_{[\mu \nu \rho]}^{a}=-\mathcal{F}_{[\mu \nu} e_{\rho]}^{a},
$$

with the abelian field strength $\mathcal{F}_{\mu \nu}=2 \partial_{[\mu} \mathcal{A}_{\nu]}$. For later supergravity calculations we also note the relation

$$
\widehat{\mathcal{R}}_{\mu \nu}{ }^{a b} \gamma^{\rho \mu \nu} \gamma_{a b}=4\left(\widehat{\mathcal{R}}^{(\rho \mu)}-\frac{1}{2} g^{\rho \mu} \widehat{\mathcal{R}}\right) \gamma_{\mu}-2(D-3) \gamma^{\rho \mu \nu} \mathcal{F}_{\mu \nu}-2(D-2) \mathcal{F}^{\rho \mu} \gamma_{\mu},
$$

with $\gamma$-matrices in $D$ space-time dimensions, and the Ricci tensor $\widehat{\mathcal{R}}_{\mu \nu}=\widehat{\mathcal{R}}_{\mu \rho \nu}{ }^{b} e_{b}{ }^{\rho}$ and Ricci scalar $\widehat{\mathcal{R}}=g^{\mu \nu} \widehat{\mathcal{R}}_{\mu \nu}$. Explicitly, the latter are given by 4

$$
\begin{aligned}
\widehat{\mathcal{R}}_{(\mu \nu)} & =R_{\mu \nu}+(D-2)\left(\nabla_{(\mu} \mathcal{A}_{\nu)}+\mathcal{A}_{\mu} \mathcal{A}_{\nu}\right)+g_{\mu \nu}\left(\nabla^{\lambda} \mathcal{A}_{\lambda}-(D-2) A^{\lambda} A_{\lambda}\right), \\
\widehat{\mathcal{R}} & =R+2(D-1) g^{\lambda \rho} \nabla_{\lambda} \mathcal{A}_{\rho}-(D-1)(D-2) \mathcal{A}_{\lambda} \mathcal{A}^{\lambda} .
\end{aligned}
$$

The covariantized Einstein equations (in absence of matter) thus are

$$
\widehat{\mathcal{R}}_{(\mu \nu)}-\frac{1}{2} \widehat{\mathcal{R}} g_{\mu \nu}=0,
$$

and manifestly invariant under (2.9). In the theories we will consider, additional matter will always be present, in particular a gauge field sector which includes the vector field $\mathcal{A}_{\mu}$, such that the right-hand side of the Einstein equations will be non-vanishing, see e.g. equation (4.41) below. One may verify that equations (2.13) do no longer descend from a standard action which is expected since we have gauged a symmetry that was not off-shell realized.

In the following we will extend the gauging to the remaining matter fields of supergravity. In particular, we need to identify among the supergravity gauge fields the vector field $\mathcal{A}_{\mu}$ used in all covariant derivatives.

\footnotetext{
${ }^{3}$ The equivalence breaks down once we consider additional matter in the theory.

${ }^{4}$ Our notation here is such that $\nabla_{\mu}$ in these equations refers to the covariant derivative $\nabla(\Gamma)_{\mu}$ in presence of the standard (non-covariantized) Christoffel symbols $\Gamma_{\mu \nu}^{\lambda}$.
} 


\subsection{Embedding tensor}

Extending the gauging to the full theory is conveniently described by resorting to the group-theoretical formalism developed in [8, 9, 10, 11]. As we have discussed in the introduction, the full global symmetry group of the ungauged theory is given by the direct product $\mathbb{R}^{+} \times \mathrm{G}$ where the first factor describes the scaling (2.1), (2.2) and the second factor is the standard duality group. We will denote the total set of generators by $\left\{t_{\hat{\alpha}}\right\}=\left\{t_{0}, t_{\alpha}\right\}, \hat{\alpha}=0, \ldots, \operatorname{dim} \mathrm{G}$, where $t_{0}$ denotes the generator of $\mathbb{R}^{+}$and the $t_{\alpha}$ denote the generators of $\mathrm{G}$. The latter satisfy commutation relations

$$
\left[t_{\alpha}, t_{\beta}\right]=f_{\alpha \beta}^{\gamma} t_{\gamma} .
$$

The vector fields $A_{\mu}^{M}$ in the ungauged theory transform in some representation $\mathcal{R}_{\mathrm{v}}$ of G labeled by $M=1, \ldots, \operatorname{dim} \mathcal{R}_{\mathrm{v}}$, and carry charge +1 under $\mathbb{R}^{+}$according to (2.1).

A general gauging is defined by introducing covariant derivatives

$$
D_{\mu} \equiv \partial_{\mu}-g A_{\mu}^{M} \widehat{\Theta}_{M}{ }^{\hat{\alpha}} t_{\hat{\alpha}}=\partial_{\mu}-g A_{\mu}^{M} \widehat{\Theta}_{M}{ }^{0} t_{0}-g A_{\mu}^{M} \widehat{\Theta}_{M}{ }^{\alpha} t_{\alpha},
$$

in terms of an embedding tensor $\widehat{\Theta}_{M}^{\hat{\alpha}}$ which describes the embedding of the gauge group generators $X_{M} \equiv \widehat{\Theta}_{M}{ }^{\hat{\alpha}} t_{\hat{\alpha}}$ into the symmetry group of the ungauged theory. In addition, we have introduced the gauge coupling constant $g$. It can in principle be absorbed into the embedding tensor.

According to its coupling the embedding tensor carries charge -1 under $\mathbb{R}^{+}$. Its component $\theta_{M} \equiv \widehat{\Theta}_{M}{ }^{0}$ transforms under $\mathrm{G}$ in the representation $\mathcal{R}_{\mathrm{v} *}$ dual to $\mathcal{R}_{\mathrm{v}}$. It selects the vector field that gauges the $\mathbb{R}^{+}$-symmetry. Comparing (2.15) to (2.4) we identify

$$
\mathcal{A}_{\mu}=g \theta_{M} A_{\mu}^{M} .
$$

The remaining part of the connection (2.15) involves the generators of the duality group $\mathrm{G}$ and is thus invisible in the gravity sector discussed above. It is defined by the component $\widehat{\Theta}_{M}{ }^{\alpha}$ of the embedding tensor which a priori transforms in the tensor product

$$
\mathcal{R}_{\mathrm{v} *} \otimes \mathcal{R}_{\text {adj }}=\mathcal{R}_{\mathrm{v} *} \oplus \ldots
$$

with $\mathcal{R}_{\text {adj }}$ denoting the adjoint representation of $\mathrm{G}$. For gaugings that do not involve the scaling symmetry $\mathbb{R}^{+}$, it is known that supersymmetry restricts the allowed choices for $\widehat{\Theta}_{M}{ }^{\alpha}$ to only very few of the irreducible representations on the r.h.s. of (2.17), see e.g. [9]. In particular, in the maximal theories (for $3 \leq D \leq 7$ ), the "trace part" $\mathcal{R}_{\mathrm{v} *}$ in this tensor product is always forbidden 5 In $D=2$ space-time dimensions on the other hand, the picture is quite the opposite: the gaugings are precisely parametrized by the "trace

\footnotetext{
${ }^{5}$ In the half-maximal theories, the representation $\mathcal{R}_{\mathrm{v} *}$ appears with multiplicity 2 on the r.h.s. of (2.17) and supersymmetry implies a linear relation between these two representations [30. The same happens for the maximal theories in $D=8,9$, where the group $\mathrm{G}$ is no longer simple [20].
} 
part" $\mathcal{R}_{\mathrm{v} *}$ on the r.h.s. of (2.17), i.e. gaugings are described by a vector $\theta_{M}$ transforming in the basic representation of the infinite-dimensional affine algebra $E_{9(9)}$ [25]. From a unifying point of view of the gauged supergravities this is somewhat unsatisfactory; upon dimensional reduction the algebraic structures in higher dimensions are typically embedded into the lower-dimensional structures described by higher-rank Kac-Moody algebras. We will see that precisely the theories that involve a gauging of the trombone symmetry close this gap and allow for a non-vanishing $\mathcal{R}_{\mathrm{v} *}$ in (2.17) also in dimensions $D>2$. Recall that only in $D=2$ dimensions, the trombone symmetry is part of the off-shell symmetries of the action and shows up as the central extension of the affine algebra $\mathrm{E}_{9(9)}$.

Our general ansatz for the embedding tensor and thus for the connection in (2.15) is the following:

$$
\widehat{\Theta}_{M}^{0}=\theta_{M}, \quad \widehat{\Theta}_{M}^{\alpha}=\Theta_{M}^{\alpha}+\zeta \theta_{N}\left(t^{\alpha}\right)_{M}{ }^{N}
$$

where we have split $\widehat{\Theta}_{M}^{\alpha}$ into a "traceless" part satisfying $\Theta_{M}{ }^{\alpha}\left(t_{\alpha}\right)_{N}{ }^{M}=0$ and its "trace part", corresponding to the representation $\mathcal{R}_{\mathrm{v} *}$ on the r.h.s. of (2.17) 6 As this second term is forbidden in the standard gaugings, it is natural to assume that it comes proportional to the same vector $\theta_{M}$ that triggers the gauging of the trombone symmetry via $\widehat{\Theta}_{M}{ }^{0}$. We will explicitly verify this assumption. All that remains in this ansatz is to determine the proportionality constant $\zeta$, which must be done case by case, i.e. in dependence of the space-time dimension and the number of supercharges.

To this end, we recall that a generic gauging introduces non-trivial couplings between vector fields and the antisymmetric two-form tensors. E.g. the non-abelian field strength of the vector fields receives corrections of the Stückelberg type [10, 11]

$$
\mathcal{F}_{\mu \nu}^{M} \longrightarrow \mathcal{H}_{\mu \nu}^{M} \equiv \mathcal{F}_{\mu \nu}^{M}+g Z_{P Q}^{M} B_{\mu \nu}^{P Q}
$$

with two-forms $B_{\mu \nu}^{P Q}$ and the intertwining tensor given by

$$
Z_{P Q}^{M} \equiv\left(t_{\hat{\alpha}}\right)_{(P}{ }^{M} \widehat{\Theta}_{Q)}{ }^{\hat{\alpha}} .
$$

In particular, this tensor encodes the field content of two-forms in the theory: as in general in its indices $P Q$ it does not project onto the full symmetric tensor product $\mathcal{R}_{\mathrm{v}} \otimes \mathcal{R}_{\mathrm{v}}$, but rather satisfies some non-trivial projection

$$
Z_{P Q}^{M}=Z_{R S}^{M} \mathbb{P}_{P Q}^{R S}
$$

also the two-forms will only appear under projection with $\mathbb{P}$, see [11, 31] for details. We will take this projection as a guide to determine the constant $\zeta$ in (2.18): the projector $\mathbb{P}$ in (2.21) and thus the two-form field content should be the same in presence and

${ }^{6}$ Here and in the following, we raise and lower adjoint indices with the invariant metric $\kappa_{\alpha \beta} \equiv$ $\operatorname{Tr}\left[t_{\alpha} t_{\beta}\right]$ which is related to the Cartan-Killing form $\eta_{\alpha \beta}$ as $\kappa_{\alpha \beta}=C_{\mathrm{v}}\left(\operatorname{dim} \mathcal{R}_{\mathrm{v}}\right) /\left(\operatorname{dim} \mathcal{R}_{\text {adj }}\right) \eta_{\alpha \beta}$ with the Casimir operator $C_{\mathrm{v}}$ in the vector field representation. 
in absence of an $\mathbb{R}^{+}$-gauging. In particular, this is necessary because the two-form field content of the theory is fixed by supersymmetry. We will further illustrate this argument and the calculation in section 3 in several examples. Later in section 4 we also explicitly confirm consistency of the ansatz (2.18) with supersymmetry.

\subsection{Quadratic constraints}

Before applying the above construction to various theories, let us collect a few general formulae and relations. With the ansatz (2.18) for the embedding tensor, the generators of the gauge group evaluated in the vector field representation are given by

$$
\left(X_{M}\right)_{N}{ }^{K} \equiv \widehat{\Theta}_{M}{ }^{\hat{\alpha}}\left(t_{\hat{\alpha}}\right)_{N}{ }^{K}=\Theta_{M}{ }^{\alpha}\left(t_{\alpha}\right)_{N}{ }^{K}+\left(\zeta\left(t^{\alpha}\right)_{M}{ }^{P}\left(t_{\alpha}\right)_{N}{ }^{K}-\delta_{M}^{P} \delta_{N}^{K}\right) \theta_{P} .
$$

In particular, this gives an explicit expression for the intertwining tensor $Z$ from (2.20)

$$
Z_{P Q}^{M}=\left(t_{\alpha}\right)_{(P}{ }^{M} \Theta_{Q)}{ }^{\alpha}+\left(\zeta\left(t^{\alpha}\right)_{P}{ }^{(K}\left(t_{\alpha}\right)_{Q}{ }^{L)}-\delta_{P}^{(K} \delta_{Q}^{L)}\right) \theta_{L},
$$

from which we will determine the values of $\zeta$ in the examples below. In every particular theory, supersymmetry will constrain the possible form of $\Theta_{M}{ }^{\alpha}$, this gives rise to the so-called linear representation constraints on the embedding tensor. In addition, consistency requires the components $\theta_{M}, \Theta_{M}{ }^{\alpha}$ of the embedding tensor to satisfy a set of rather generic quadratic constraints. These express the fact that the embedding tensor itself is invariant under the action of the gauge group. Evaluating this condition for the different components gives rise to the equations

$$
\begin{aligned}
0 \stackrel{!}{\equiv} \delta_{M} \theta_{N} & =\widehat{\Theta}_{M}^{\hat{\alpha}} \delta_{\hat{\alpha}} \theta_{N} \\
& =\left(t_{\gamma}\right)_{N}{ }^{Q} \Theta_{M}{ }^{\gamma} \theta_{Q}+\left(\zeta\left(t^{\gamma}\right)_{M}{ }^{K}\left(t_{\gamma}\right)_{N}{ }^{L}-\delta_{M}^{K} \delta_{N}^{L}\right) \theta_{K} \theta_{L}
\end{aligned}
$$

and

$$
\begin{aligned}
0 \stackrel{!}{\equiv} \delta_{P} \Theta_{M}^{\alpha}= & \Theta_{P}^{\beta}\left(t_{\beta}\right)_{M}{ }^{N} \Theta_{N}{ }^{\alpha}+\Theta_{P}{ }^{\beta} f_{\beta \gamma}{ }^{\alpha} \Theta_{M}{ }^{\gamma} \\
& +\left(\zeta \delta_{\beta}^{\alpha}\left(t^{\gamma}\right)_{P}{ }^{Q}\left(t_{\gamma}\right)_{M}{ }^{N}-\zeta f_{\beta \gamma}{ }^{\alpha} \delta_{M}^{N}\left(t^{\gamma}\right)_{P}{ }^{Q}-\delta_{P}^{Q} \delta_{M}^{N} \delta_{\beta}^{\alpha}\right) \theta_{Q} \Theta_{N}{ }^{\beta}
\end{aligned}
$$

Together, they guarantee in particular that the gauge group generators (2.22) satisfy

$$
\left[X_{M}, X_{N}\right]=-\left(X_{M}\right)_{N}{ }^{K} X_{K}
$$

i.e. these constraints ensure closure of the gauge algebra. We will show in the following for various theories that every set of $\theta_{M}, \Theta_{M}{ }^{\alpha}$ that satisfies the given linear representation constraints and the quadratic relations (2.24), (2.25) defines a consistent gauging which in case $\theta_{M} \neq 0$ involves a gauging of the trombone symmetry.

Let us finally note that the quadratic constraints (2.24), (2.25) in particular imply the relations

$$
\theta_{M} Z_{P Q}^{M}=0=\widehat{\Theta}_{M}{ }^{\alpha} Z_{P Q}^{M},
$$

i.e. orthogonality between the embedding tensor and the intertwining tensor $Z$ from (2.23). This plays an important role in the hierarchy of antisymmetric $p$-forms [11, 31]. 


\section{Algebraic structure in various dimensions}

In this section, we work out the above construction for the maximal supergravities in dimensions $6 \geq D \geq 3$, for which the global symmetry groups are given by $\mathrm{SO}(5,5)$, $\mathrm{E}_{6(6)}, \mathrm{E}_{7(7)}$, and $\mathrm{E}_{8(8)}$, respectively. We determine for the various cases the value of the parameter $\zeta$ in (2.18), which completely fixes the algebraic structure. Furthermore, we evaluate the quadratic constraints (2.24), (2.25) and show that they admit non-trivial solutions. As a result we obtain the full set of consistency constraints for gaugings that involve a local trombone symmetry (1.1).

We will discuss in most detail the case $D=6$ in which the symmetry group is the smallest and accordingly the algebraic structures are the simplest ones. Subsequently, we report the results in lower dimensions which are obtained in complete analogy with slightly bigger computational effort.

\section{1 $D=6$}

The ungauged theory in $D=6$ dimensions was constructed in 32], its general gaugings were given in [26], to which we refer for details of the structure. The global symmetry group of the ungauged theory is the orthogonal group $\mathrm{SO}(5,5)$. Vector and two-form fields of this theory transform in the $\mathbf{1 6}_{c}$ and $\mathbf{1 0}$ representations, respectively, with the generators $t_{\alpha}=t_{[i j]}$ given by

$$
\left(t_{i j}\right)_{M^{N}}=\left(\gamma_{i j}\right)_{M}^{N}, \quad\left(t_{i j}\right)_{k}^{l}=4 \eta_{k[i} \delta_{j]}^{l},
$$

respectively. Here, $i, j, \ldots=1, \ldots, 10$, and $M, N=1, \ldots, 16$, label the vector and the spinor representation of $\mathrm{SO}(5,5)$, respectively. The tensors $\eta_{i j}$ and $\left(\gamma_{i j}\right)_{M}{ }^{N}$ denote the invariant form and the gamma matrices of $\mathrm{SO}(5,5)$, respectively. We use the former to raise and lower vector indices. A non-trivial relation among the generators that we will exploit in the following, is 7

$$
\left(t^{\alpha}\right)_{M}{ }^{K}\left(t_{\alpha}\right)_{N}{ }^{L}=-\frac{1}{32}\left(\gamma^{i j}\right)_{M}{ }^{K}\left(\gamma_{i j}\right)_{N}{ }^{L}=\frac{1}{16} \delta_{M}^{K} \delta_{N}^{L}+\frac{1}{4} \delta_{M}^{L} \delta_{N}^{K}-\frac{1}{8}\left(\gamma^{i}\right)_{M N}\left(\gamma_{i}\right)^{K L},
$$

which can be proven by further contraction with gamma matrices.

The embedding tensor $\widehat{\Theta}_{M}^{\alpha}$ describing the generators within the duality group $\mathrm{SO}(5,5)$ a priori lives in the tensor product

$$
16_{s} \otimes 45=16_{s} \oplus 144_{c} \oplus 560_{\mathrm{s}},
$$

where 45 is the adjoint of $\mathrm{SO}(5,5)$. In absence of the $\mathbb{R}^{+}$-gauging, supersymmetry restricts the embedding tensor $\Theta_{M}{ }^{\alpha}$ to the irreducible $\mathbf{1 4 4}$ representation in this decomposition [9, 26]. I.e. it can be parametrized in terms of a gamma-traceless vectorspinor $\theta^{M i}$ as

$$
\Theta_{M}^{i j}=-\theta^{N[i} \gamma^{j]}{ }_{N M}, \quad \text { with } \quad \gamma_{i M N} \theta^{N i} \equiv 0
$$

${ }^{7}$ As mentioned above, adjoint indices are raised and lowered with the invariant form $\kappa_{\alpha \beta} \equiv \operatorname{Tr}\left[t_{\alpha} t_{\beta}\right]$ which here is given by $\kappa_{i j, k l}=-32 \delta_{i[k} \delta_{l] j}$. 
In this case, the intertwining tensor (2.20) is given by

$$
Z_{P Q}^{M}=-\theta^{L i} \gamma_{L(P}^{j}\left(\gamma_{i j}\right)_{Q)}{ }^{M}=-\left(\gamma_{i}\right)_{P Q} \theta^{M i}
$$

where we have used the properties of the $\mathrm{SO}(5,5)$ gamma matrices. The form of (3.5) shows that in indices $(P Q)$, this tensor projects onto a subrepresentation

$$
\left(16_{c} \otimes 16_{c}\right)_{\mathrm{sym}} \longrightarrow 10
$$

within the full symmetric tensor product. According to the general discussion above, this must reproduce the field content of the two-forms of the theory. E.g. the general coupling (2.19) reduces to

$$
\mathcal{H}_{\mu \nu}^{M}=\mathcal{F}_{\mu \nu}^{M}-g \theta^{M i} B_{\mu \nu i},
$$

with two-forms $B_{\mu \nu i} \equiv\left(\gamma_{i}\right)_{P Q} B_{\mu \nu}^{P Q}$ transforming in the 10. Indeed, this precisely coincides with the field content of the ungauged theory. Remarkably, this gives a purely bosonic justification of the restriction of the embedding tensor within (3.3). Any other component in $\Theta_{M}{ }^{\alpha}$ would have required a larger set of two-forms and thus be in conflict with the field content of the theory (which in turn is determined by supersymmetry).

Let us now repeat this analysis in presence of an $\mathbb{R}^{+}$-gauging, i.e. for non-vanishing tensor $\theta_{M}$. In this case, the gauge group generators (2.22) are given by

$$
X_{M N}{ }^{K}=-\theta^{L i} \gamma^{j}{ }_{L M}\left(\gamma_{i j}\right)_{N}{ }^{K}-\frac{1}{32} \zeta\left(\gamma^{i j}\right)_{M}{ }^{L}\left(\gamma_{i j}\right)_{N}{ }^{K} \theta_{L}-\theta_{M} \delta_{N}^{K} .
$$

Using (3.2), we obtain for the intertwining tensor $Z$ of $(\underline{2.23})$

$$
Z_{P Q}^{M}=-\left(\gamma_{i}\right)_{P Q}\left(\theta^{M i}+\frac{\zeta}{8}\left(\gamma^{i}\right)^{M L} \theta_{L}\right)+\left(\frac{5 \zeta}{16}-1\right) \delta_{(P}^{M} \theta_{Q)}
$$

Comparing this tensor to (3.5) shows that choosing $\zeta=16 / 5, Z$ projects onto the same subspace (3.6) as in absence of the $\mathbb{R}^{+}$-gauging

$$
Z_{P Q}^{M}=\left(\gamma_{i}\right)_{P Q} \hat{Z}^{M i}, \quad \hat{Z}^{K i} \equiv-\theta^{K i}-\frac{2}{5}\left(\gamma^{i}\right)^{K L} \theta_{L} .
$$

Any other value of $\zeta$ would require a larger set of two-forms for consistency of the gauged theory and thus eventually be inconsistent with supersymmetry.

To summarize, we have found that the presence of a $\mathbf{1 6}$ component in the embedding tensor (3.3) is possible, if simultaneously the $\mathbb{R}^{+}$trombone symmetry is gauged. The explicit ansatz for the gauge group generators is given by (3.8) with $\zeta=16 / 5$. This finishes the discussion of the linear representation constraint satisfied by the embedding tensor in presence of an $\mathbb{R}^{+}$-gauging.

It remains to evaluate the quadratic constraints (2.24), (2.25) required for consistency of the gauging. The constraints (2.24) split into

$$
\left(\gamma_{i}\right)^{K[M} \theta^{N] i} \theta_{K} \stackrel{!}{\equiv} 0, \quad \theta^{K i} \theta_{K} \stackrel{!}{\equiv}-\frac{2}{5}\left(\gamma^{i}\right)^{K L} \theta_{K} \theta_{L},
$$


in terms of the irreducible components $\theta^{K i}$ and $\theta_{K}$. These constraints, which are automatically satisfied for $\theta_{K}=0$ transform under $\operatorname{SO}(5,5)$ in the $\mathbf{1 2 0}$ and the $\mathbf{1 0}$ representation, respectively. Note that the part transforming in the $\mathbf{1 2 6}_{s}+\mathbf{1 2 6}_{c}$ which could in principle be present in (2.24) is absent in (3.11), thanks to the particular choice of $\zeta$. This is crucial for the existence of non-trivial solutions. Some computation shows that the remaining quadratic constraints (2.25) may be cast into the form

$$
\begin{aligned}
\theta^{M m} \theta_{m}^{N} & \stackrel{!}{\equiv}\left(\gamma^{m}\right)^{K(M} \theta_{m}^{N)} \theta_{K}+\frac{1}{5} \gamma_{m}^{M N} \theta^{K i} \theta_{K} \\
\theta^{M i} \theta^{N[k}\left(\gamma^{l]}\right)_{M N} & \stackrel{!}{=} \frac{1}{10}\left(\gamma^{k l}\right)_{M}{ }^{N} \theta^{M i} \theta_{N}-\frac{3}{5} \theta_{M} \theta^{M[k} \eta^{l] i}
\end{aligned}
$$

transforming in the $\mathbf{1 0} \oplus \mathbf{1 2 6}_{c} \oplus \mathbf{3 2 0}$ of $\mathrm{SO}(5,5)$ and showing explicitly how the known quadratic constraints of [26] are modified by the presence of a non-vanishing $\theta_{M}$.

Every solution $\theta_{M}, \theta^{M i}$ of the combined set of quadratic constraints (3.11), (3.12) will give rise to a consistent gauging of the maximal supergravity in $D=6$. We have shown that this complete set of constraints transforms as

$$
\mathcal{R}_{\text {quad }}=\left(10 \oplus \mathbf{1 2 6}_{c} \oplus \mathbf{3 2 0}\right) \oplus(\mathbf{1 0} \oplus \mathbf{1 2 0})
$$

of which the last two representations correspond to (3.11) and are only relevant for a non-vanishing $\theta_{M}$. An important non-trivial result in this computation (which again hinges on the particular value of $\zeta=16 / 5$ in (2.18) determined above) is the absence of the $\mathbf{1 7 2 8}$ representation in (3.12) which is a priori possible in (2.25). As it constitutes the major part of the tensor product $\theta^{M i} \otimes \theta_{K}$, a mixed constraint in this representation would presumably exclude any solution with both $\theta^{M i}$ and $\theta_{K}$ non-vanishing. Instead, we expect a rather rich class of solutions of the quadratic constraints (3.11), (3.12) with simultaneously non-vanishing $\theta^{M i}$ and $\theta_{K}$. We leave the study of such theories to future work.

Let us analyze here in detail the subclass of gaugings with $\theta^{M i}=0$, which are thus complementary to the gaugings studied in [26]. These theories are parametrized by an $\mathrm{SO}(5,5)$ spinor $\theta_{M}$ for which the constraints (3.11) reduce to

$$
\left(\gamma_{i}\right)^{K L} \theta_{K} \theta_{L} \equiv 0
$$

Funny enough, this is precisely the structure of an $\mathrm{SO}(10)$ pure spinor (albeit for a different real form than the usual $\mathrm{SO}(1,9)$ ) that shows up in a very different context here - classifying a particular subsector of possible gaugings in maximal six-dimensional supergravity. We can use this to employ the well-known parametrization of the general solution of this quadratic constraint upon decomposing $\theta_{M}$ into its GL(5)-irreducible parts $\left(\xi, \xi^{m}, \xi_{[m n]}\right)$ with $m, n=1, \ldots, 5$, according to the branching

$$
\mathbf{1 6}_{s} \longrightarrow 1^{-5} \oplus 5^{\prime+3} \oplus 10^{-1}
$$

of $\mathrm{SO}(5,5)$ under $\mathrm{GL}(5)$. In terms of these components, the quadratic constraint (alias the pure spinor condition (3.14)) decomposes into the conditions

$$
\xi \xi^{m}=\epsilon^{m k l p q} \xi_{k l} \xi_{p q}, \quad \xi^{m} \xi_{m n}=0
$$


with the totally antisymmetric tensor $\epsilon^{m k l p q}$. On a patch with $\xi \neq 0$, these equations are simultaneously solved by setting

$$
\xi^{m}=\epsilon^{m k l p q} \xi_{k l} \xi_{p q} / \xi,
$$

leaving 11 independent real parameters $\left(\xi, \xi_{m n}\right)$ in the general solution.

We have thus found a particular class of maximal supersymmetric gaugings defined by $\theta_{M}=\left(\xi, \xi^{m}, \xi_{[m n]}\right)$ with $\xi^{m}$ given in (3.17). Moreover, this is the most general gauging with the components $\theta^{M i}=0$. As GL(5) is the global symmetry group of sevendimensional maximal supergravity, it is tempting to speculate that these theories have a possible higher-dimensional origin as particular (generalized) circle compactifications from seven dimensions. In the following we will see that a very similar pattern shows up for the analogous class of gaugings in lower dimensions.

\section{$3.2 D=5$}

The ungauged theory in $D=5$ dimensions was constructed in [33], its general gaugings were given in [10]. The global symmetry group of the ungauged theory is $\mathrm{E}_{6(6)}$. Vector and two-form fields of this theory transform in the mutually dual $\overline{\mathbf{2 7}}$ and $\mathbf{2 7}$ representation. In the ungauged theory, only the vector fields appear in the Lagrangian while the two-forms are defined as their on-shell duals. A non-trivial relation among the $\mathrm{E}_{6(6)}$ generators that we will exploit in the following, is

$$
\left(t^{\alpha}\right)_{M}^{K}\left(t_{\alpha}\right)_{N}^{L}=\frac{1}{18} \delta_{M}^{K} \delta_{N}^{L}+\frac{1}{6} \delta_{M}^{L} \delta_{N}^{K}-\frac{5}{3} d_{M N P} d^{K L P}
$$

where $d_{M N K}$ and $d^{M N K}$ are the totally symmetric $\mathrm{E}_{6(6)}$ invariant tensors, normalized as $d_{M N K} d^{M N L}=\delta_{K}^{L}$, see [10] for further useful relations.

The embedding tensor $\widehat{\Theta}_{M}{ }^{\alpha}$ a priori lives in the tensor product

$$
27 \otimes 78=27 \oplus \overline{351} \oplus 1728
$$

where $\mathbf{7 8}$ is the adjoint of $\mathrm{E}_{6(6)}$. In absence of the $\mathbb{R}^{+}$-gauging, supersymmetry restricts the embedding tensor $\Theta_{M}{ }^{\alpha}$ to the $\overline{\mathbf{3 5 1}}$ representation in this decomposition [9, 10]. I.e. it can be parametrized in terms of an antisymmetric matrix $Z^{M N}=-Z^{N M}$ as

$$
\Theta_{M}{ }^{\alpha}=12 Z^{P Q}\left(t^{\alpha}\right)_{R}^{S} d^{R K L} d_{M P K} d_{S Q L} .
$$

Using (3.18), we obtain for the full intertwining tensor $Z$ of (2.23)

$$
Z_{P Q}^{M}=d_{P Q L}\left(Z^{M L}-\frac{5 \zeta}{3} d^{M L Q} \theta_{Q}\right)+\left(\frac{2 \zeta}{9}-1\right) \delta_{(P}^{M} \theta_{Q)} .
$$

This shows that choosing $\zeta=9 / 2$, this tensor simplifies to

$$
Z_{P Q}^{M}=d_{P Q L}\left(Z^{M L}-\frac{15}{2} d^{M L Q} \theta_{Q}\right) \equiv d_{P Q L} \widehat{Z}^{M L},
$$

and thus projects onto a single subrepresentation

$$
(\overline{\mathbf{2 7}} \otimes \overline{\mathbf{2 7}})_{\mathrm{sym}} \longrightarrow 27,
$$


within the full symmetric tensor product, and thus onto the same subspace as in absence of the $\mathbb{R}^{+}$-gauging. This is precisely compatible with the two-forms present in the theory. Any other value of $\zeta$ would for consistency require a larger set of two-forms and thus be incompatible with supersymmetry.

It is interesting to note, that in absence of the $\mathbb{R}^{+}$-gauging, the tensor $\widehat{Z}^{M N}$ of (3.22) is totally antisymmetric and as such also shows up in the topological coupling of the two-forms in the action $\mathcal{L}_{B d B}=Z^{M N} B_{M} \wedge d B_{N}$. The fact that for non-vanishing $\theta_{K}$ this tensor is no longer antisymmetric reflects the fact that the $\mathbb{R}^{+}$-gaugings in general do no longer admit an action.

It remains to evaluate the quadratic constraints (2.24), (2.25) required for consistency of the gauging. The quadratic constraints (2.24) split into

$$
Z^{P Q} \theta_{R} d_{P K M} d_{Q L N} d^{K L R} \stackrel{!}{\equiv} 0, \quad Z^{M N} \theta_{N} \stackrel{!}{\equiv} 15 d^{M K L} \theta_{K} \theta_{L}
$$

in terms of the irreducible components $Z^{M N}$ and $\theta_{K}$. These constraints, which are automatically satisfied for $\theta_{M}=0$ transform under $\mathrm{E}_{6(6)}$ in the $\mathbf{3 5 1}$ and the $\overline{\mathbf{2 7}}$, respectively. After some computation, the quadratic constraints (2.25) take the form

$$
4\left(t_{\alpha}\right)_{K}^{L} Z^{K R} Z^{N S} d_{R S L}+3\left(t_{\alpha}\right)_{K}^{L} Z^{K N} \theta_{L}+3\left(t_{\alpha}\right)_{K}{ }^{N} Z^{K L} \theta_{L} \stackrel{!}{\equiv} 0 .
$$

The first term transforms under $\mathrm{E}_{6(6)}$ in the $\overline{\mathbf{2 7}}+\overline{\mathbf{1 7 2 8}}$ [10], and the form of (3.25) shows that the additional terms (upon imposing (3.24)) fall into the same representations. I.e. the total quadratic constraint transforms as

$$
\mathcal{R}_{\text {quad }}=(\overline{\mathbf{2 7}} \oplus \overline{\mathbf{1 7 2 8}}) \oplus(\overline{\mathbf{2 7}} \oplus \mathbf{3 5 1})
$$

of which the last two representations correspond to (3.24) and are only relevant in presence of an $\mathbb{R}^{+}$-gauging. An important non-trivial result in this constraint analysis (which again hinges on the particular value of $\zeta=9 / 2$ in (2.18)) is the absence of the $\overline{\mathbf{7 3 7 1}}$ representation which is a priori possible in (3.25). As it constitutes the major part of the tensor product $Z^{M N} \otimes \theta_{K}$, its presence among the constraints would presumably exclude any solution with both $Z^{M N}$ and $\theta_{K}$ non-vanishing.

Let us finally discuss the particular gaugings for which $Z^{M N}=0$ and which are thus complementary to those constructed in [10]. In this case, the only non-trivial quadratic constraint on the remaining component $\theta_{K}$ comes from (3.24) and is given by

$$
d^{M K L} \theta_{K} \theta_{L} \stackrel{!}{\equiv} 0
$$

This condition can be viewed as the "analogue of a pure spinor" (3.14) for the exceptional group $\mathrm{E}_{6(6)}$. We can employ a similar technique to explicitly solve it. To this end, we decompose $\theta_{M}$ into its $\left(\mathrm{SO}(5,5) \times \mathbb{R}^{+}\right)$-irreducible parts $\left(\lambda, \lambda_{i}, \lambda_{\alpha}\right)$ according to the branching

$$
27 \longrightarrow 1^{+4} \oplus 10^{-2} \oplus 16_{s}^{+1}
$$


The quadratic constraint (3.27) accordingly decomposes into the equations

$$
\lambda \lambda_{i}=\left(\gamma_{i}\right)^{\alpha \beta} \lambda_{\alpha} \lambda_{\beta}, \quad\left(\gamma^{i}\right)^{\alpha \beta} \lambda_{i} \lambda_{\beta}=0, \quad \lambda_{i} \lambda^{i}=0,
$$

with the $\mathrm{SO}(5,5)$ tensors $\left(\gamma_{i}\right)^{\alpha \beta}$ introduced in the last subsection 8 On a patch where $\lambda \neq 0$, these equations are simultaneously solved by setting

$$
\lambda_{i}=\left(\gamma_{i}\right)^{\alpha \beta} \lambda_{\alpha} \lambda_{\beta} / \lambda
$$

This is straightforwardly verified using the well-known identity $\left(\gamma^{i}\right)^{(\alpha \beta}\left(\gamma_{i}\right)^{\gamma) \delta}=0$ for $\mathrm{SO}(5,5)$ gamma-matrices. In total, this leaves 17 independent real parameters $\left(\lambda, \lambda_{\alpha}\right)$ for the general solution of (3.27).

\section{3 $D=4$}

The ungauged theory in $D=4$ dimensions was constructed in [2], its general gaugings were given in [24]. The global symmetry group of the ungauged theory is $\mathrm{E}_{7(7)}$. Vector and two-form fields of this theory transform in the $\mathbf{5 6}$ and the adjoint $\mathbf{1 3 3}$ representations, respectively. In the ungauged theory, only 28 electric vector fields appear in the Lagrangian while their 28 magnetic duals are defined on-shell. Similarly, the two-forms are defined on-shell as duals to the scalar fields of the theory.

A non-trivial relation among the $\mathrm{E}_{7(7)}$ generators that we will exploit in the following, is

$$
\left(t^{\alpha}\right)_{M}^{K}\left(t_{\alpha}\right)_{N}^{L}=\frac{1}{24} \delta_{M}^{K} \delta_{N}^{L}+\frac{1}{12} \delta_{M}^{L} \delta_{N}^{K}+\left(t^{\alpha}\right)_{M N}\left(t_{\alpha}\right)^{K L}-\frac{1}{24} \Omega_{M N} \Omega^{K L},
$$

where the fundamental indices have been raised and lowered with the symplectic matrix $\Omega_{M N}$ (and we use north-west south-east conventions, i.e. $X^{M}=\Omega^{M N} X_{N}$, etc.).

The embedding tensor $\widehat{\Theta}_{M}{ }^{\alpha}$ a priori lives in the tensor product

$$
56 \otimes 133=56 \oplus 912 \oplus 6480
$$

In absence of the $\mathbb{R}^{+}$-gauging, supersymmetry restricts the embedding tensor $\Theta_{M}{ }^{\alpha}$ to the 912 representation in this decomposition [9, 24], i.e. to a tensor satisfying the condition $\Theta_{M}{ }^{\alpha}=-2\left(t_{\beta} t^{\alpha}\right)_{M}{ }^{N} \Theta_{N}{ }^{\beta}$.

Using (3.31), we obtain for the full intertwining tensor $Z$ of (2.23)

$$
Z_{P Q}^{M}=\left(t_{\alpha}\right)_{P Q}\left(-\frac{1}{2} \Theta_{L}{ }^{\alpha} \Omega^{M L}+\zeta\left(t_{\alpha}\right)^{M L} \theta_{L}\right)+\left(\frac{\zeta}{8}-1\right) \delta_{(P}^{M} \theta_{Q)} .
$$

This shows that upon choosing $\zeta=8$, this tensor simplifies to

$$
Z_{P Q}^{M}=-\frac{1}{2}\left(t_{\alpha}\right)_{P Q}\left(\Theta^{M \alpha}-16\left(t_{\alpha}\right)^{M L} \theta_{L}\right) \equiv\left(t_{\alpha}\right)_{P Q} \widehat{Z}^{M \alpha}
$$

\footnotetext{
${ }^{8}$ In contrast to the last subsection, we here use indices $\alpha, \beta$ for the $\mathrm{SO}(5,5)$ spinor representation, as capital indices $M, N$ in this section are reserved for the $\mathrm{E}_{6(6)}$ fundamental representation of the vector fields.
} 
and thus projects onto a single subrepresentation

$$
(56 \otimes 56)_{\mathrm{sym}} \longrightarrow 133
$$

within the full symmetric tensor product, which is the same subspace as in absence of the $\mathbb{R}^{+}$-gauging. This is precisely compatible with the content of two-forms present in the theory. Any other value of $\zeta$ would for consistency require a larger set of two-forms and thus be incompatible with supersymmetry.

It remains to evaluate the quadratic constraints (2.24), (2.25) required for consistency of the gauging. The quadratic constraints (2.24) split into

$$
\left(t_{\gamma}\right)_{\left[M^{Q}\right.} \Theta_{N]}^{\gamma} \theta_{Q} \stackrel{!}{\equiv} 0, \quad \Omega^{P Q} \Theta_{P}^{\alpha} \theta_{Q} \stackrel{!}{\equiv}-16\left(t^{\alpha}\right)^{K L} \theta_{K} \theta_{L},
$$

transforming in the 1539 and the 133 of $\mathrm{E}_{7(7)}$, respectively. As in the higher dimensions discussed above, the quadratic constraint (2.25) in presence of a $\theta_{M}$ induces a modification of the known quadratic constraints [24] which is given by

$$
\Theta_{M}{ }^{\alpha} \Theta_{N}{ }^{\beta} \Omega^{M N} \stackrel{!}{\equiv} 8 \theta_{M} \Theta_{N}{ }^{[\alpha} t^{\beta] M N}-4 f_{\gamma}^{\alpha \beta} \theta_{M} \Theta_{N}^{\gamma} \Omega^{M N}
$$

Together, we find that the total set of quadratic constraints transforms under $\mathrm{E}_{7(7)}$ in the representation

$$
\mathcal{R}_{\text {quad }}=(133 \oplus 8645) \oplus(133 \oplus 1539)
$$

of which the last two representations correspond to (3.36) and are only relevant in presence of a non-vanishing $\theta_{M}$.

Let us finally discuss the particular gaugings for which $\Theta_{M}{ }^{\alpha}=0$ and which are thus complementary to those constructed previously in [24]. In this case, the only nontrivial quadratic constraint on the remaining component $\theta_{K}$ comes from (3.36) and is given by

$$
\left(t_{\alpha}\right)^{K L} \theta_{K} \theta_{L} \stackrel{!}{\equiv} 0
$$

This condition can be viewed as the "analogue of a pure spinor" (3.14) for the exceptional group $\mathrm{E}_{7(7)}$. In complete analogy to the analysis for the groups $\mathrm{SO}(5,5)$ and $\mathrm{E}_{6(6)}$ above, we can find its most general solution by decomposing $\theta_{M}$ into its $\left(\mathrm{E}_{6(6)} \times \mathbb{R}^{+}\right)$-irreducible parts $\left(\eta, \eta_{m}, \eta^{m}, \tilde{\eta}\right)$ according to the branching

$$
56 \longrightarrow 1^{-3} \oplus 27^{-1} \oplus 27^{\prime+1} \oplus 1^{+3} .
$$

The quadratic constraint (3.39) accordingly decomposes into the set of equations

$$
\eta \eta^{m}=d^{m k l} \eta_{k} \eta_{l}, \quad \tilde{\eta} \eta_{m}=d_{m k l} \eta^{k} \eta^{l}, \quad \eta \tilde{\eta}-\frac{2}{15} \eta_{m} \eta^{m}=0=\left(t_{a}\right)_{n}{ }^{m} \eta_{m} \eta^{n}
$$


with the $\mathrm{E}_{6(6)}$ tensors $d_{m n k}$ and $\mathrm{E}_{6(6)}$ generators $\left(t_{a}\right)_{n}{ }^{m}$ introduced in the last subsection 9 On a patch where $\eta \neq 0$, these equations are simultaneously solved by setting

$$
\eta^{m}=d^{m k l} \eta_{k} \eta_{l} / \eta, \quad \tilde{\eta}=\frac{2}{15} d^{p q r} \eta_{p} \eta_{q} \eta_{r} / \eta^{2}
$$

upon using the identity $d_{p(k l} d_{m n) q} d^{p q r}=\frac{2}{15} \delta_{(k}^{r} d_{l m n)}$. This leaves 28 independent parameters $\left(\eta, \eta_{m}\right)$ in the general solution 10

\section{$3.4 \quad D=3$}

The ungauged theory in $D=3$ dimensions was constructed in [35], its general gaugings were given in [8]. The global symmetry group of the ungauged theory is $\mathrm{E}_{8(8)}$. The three-dimensional theory is special in that the ungauged theory does not carry any vector fields which appear in the gauged theory via a Chern-Simons coupling. As they are dual to the scalar fields, they transform in the adjoint of $\mathrm{E}_{8(8)}$, which is the 248-dimensional representation with generators 11

$$
\left(t_{\mathcal{M}}\right)_{\mathcal{N}}^{\mathcal{K}}=-f_{\mathcal{M N}}{ }^{\mathcal{K}}
$$

in terms of the structure constants of $\mathrm{E}_{8(8)}$. Two forms transform in the $\mathbf{1} \oplus \mathbf{3 8 7 5}$ representation of $\mathrm{E}_{8(8)}$. Although these forms are non-propagating in three dimensions, their field content can be inferred from the supersymmetry algebra or from their onshell duality to the embedding tensor [31, 44].

The embedding tensor $\widehat{\Theta}_{\mathcal{M N}}$ a priori lives in the tensor product

$$
248 \otimes 248=1 \oplus 248 \oplus 3875 \oplus \mathbf{2 7 0 0 0} \oplus 30380
$$

In absence of the $\mathbb{R}^{+}$-gauging, supersymmetry restricts the embedding tensor to the reducible $\mathbf{1} \oplus \mathbf{3 8 7 5}$ representation in this decomposition [8]. Explicitly, this is a symmetric tensor $\Theta_{\mathcal{M N}}$ which satisfies

$$
\Theta_{\mathcal{M N}}=\left(\left(\mathbb{P}_{\mathbf{1}}\right)_{\mathcal{M N}}{ }^{\mathcal{K L}}+\left(\mathbb{P}_{\mathbf{3 8 7 5}}\right)_{\mathcal{M N}}{ }^{\mathcal{K}}\right) \Theta_{\mathcal{K} \mathcal{L}}
$$

with the projectors

$$
\begin{aligned}
\left(\mathbb{P}_{\mathbf{1}}\right)_{\mathcal{M N}}{ }^{\mathcal{K L}} & =\frac{1}{248} \eta_{\mathcal{M N}} \eta^{\mathcal{K L}} \\
\left(\mathbb{P}_{\mathbf{3 8 7 5}}\right)_{\mathcal{M N}}{ }^{\mathcal{K} \mathcal{L}} & =\frac{1}{7} \delta_{(\mathcal{M}}^{\mathcal{K}} \delta_{\mathcal{N})}^{\mathcal{L}}-\frac{1}{14} f^{\mathcal{P K}}{ }_{(\mathcal{M}} f_{\mathcal{N}) \mathcal{P}}{ }^{\mathcal{L}}-\frac{1}{56} \eta_{\mathcal{M N}} \eta^{\mathcal{K} \mathcal{L}} .
\end{aligned}
$$

\footnotetext{
${ }^{9}$ In contrast to the last subsection, we here use indices $m, n$ for the $\mathrm{E}_{6(6)}$ fundamental representation, as capital indices $M, N$ in this section are reserved for the $\mathrm{E}_{7(7)}$ fundamental representation of the vector fields.

${ }^{10} \mathrm{As}$ a byproduct, we thus find that an $\mathrm{E}_{7(7)}$ vector $\theta_{M}$ subject to the quadratic condition (3.39) represents a very compact way to describe the non-linear conformal realization of this group [34] on a 27-dimensional vector space.

${ }^{11}$ In order to facilitate comparison with previous work in three dimensions [8, 31, we use in this section calligraphic indices for the fundamental (=adjoint) representation. Moreover, we use the Cartan-Killing form $\eta_{\mathcal{M N}}$ rather than the rescaled form $\kappa_{\mathcal{M N}}=60 \eta_{\mathcal{M N}}$ defined in footnote 4 and used in the previous sections to raise and lower adjoint indices.
} 
Using (2.18), we obtain for the full intertwining tensor $Z$ of (2.23)

$$
Z^{\mathcal{M}}{ }_{\mathcal{P} \mathcal{Q}}=f^{\mathcal{L K}}{ }_{(\mathcal{P}} \Theta_{\mathcal{Q}) \mathcal{L}}+\left(\zeta f^{\mathcal{L} \mathcal{M}_{(\mathcal{P}} f_{\mathcal{Q}) \mathcal{L}}}{ }^{\mathcal{K}}-\delta_{(\mathcal{P}}^{\mathcal{M}} \delta_{\mathcal{Q})}^{\mathcal{K}}\right) \theta_{\mathcal{K}}
$$

With the explicit form of the projectors (3.46) this shows that choosing $\zeta=1 / 2$, the tensor $Z$ projects onto the subrepresentation

$$
(248 \otimes 248)_{\mathrm{sym}} \longrightarrow 1 \oplus 3875
$$

within the full symmetric tensor product, and thus onto the same subspace as in absence of the $\mathbb{R}^{+}$-gauging. This is precisely compatible with the two-forms present in the theory (which are dual to the embedding tensor) and thus compatible with supersymmetry as we shall explicitly demonstrate in the next section.

It is interesting to note, that in absence of the $\mathbb{R}^{+}$-gauging, the tensor $\widehat{\Theta}_{\mathcal{M N}}=\Theta_{\mathcal{M N}}$ is symmetric in its two indices and also shows up as a metric of the Chern-Simons term in the action $\mathcal{L}_{\mathrm{CS}}=\Theta_{\mathcal{M N}} A^{\mathcal{M}} \wedge d A^{\mathcal{N}}$. The fact that for non-vanishing $\theta_{\mathcal{K}}$, this tensor is no longer symmetric again reflects the fact that the $\mathbb{R}^{+}$-gaugings in general do no longer admit an action.

It remains to evaluate the quadratic constraints (2.24), (2.25) required for consistency of the gauging which yields

$$
\begin{aligned}
\theta_{\mathcal{M}} \theta_{\mathcal{N}}-\frac{1}{2} f^{\mathcal{Q P}}{ }_{\mathcal{N}} f_{\mathcal{M} \mathcal{Q}}{ }^{\mathcal{L}} \theta_{\mathcal{P}} \theta_{\mathcal{L}} & =\Theta_{\mathcal{L M}} f_{\mathcal{N}}{ }^{\mathcal{L P}} \theta_{\mathcal{P}} \\
2 \Theta_{\mathcal{M L}} \Theta_{\mathcal{T}(\mathcal{N}} f_{\mathcal{P})}{ }^{\mathcal{L T}}-\Theta_{\mathcal{N} \mathcal{P}} \theta_{\mathcal{M}}-\Theta_{\mathcal{L}(\mathcal{N}} f_{\mathcal{P})}{ }^{\mathcal{L T}} f_{\mathcal{M} \mathcal{T}}{ }^{Q_{\mathcal{Q}}} & =0
\end{aligned}
$$

In particular, contraction of these equations implies that

$$
\theta^{\mathcal{M}} \theta_{\mathcal{M}}=0, \quad \eta^{\mathcal{M N}} \Theta_{\mathcal{M N}} \theta_{\mathcal{K}}=0=\Theta_{\mathcal{K} \mathcal{M}} \theta^{\mathcal{M}}
$$

With some effort one can show that these constraints transform in the

$$
\mathcal{R}_{\text {quad }}=(3875 \oplus \mathbf{1 4 7 2 5 0}) \oplus(1 \oplus 2 \cdot 248 \oplus 3875 \oplus \mathbf{3 0 3 8 0})
$$

of which the last four representations correspond to (3.49) and (3.51) and are only relevant for a non-vanishing $\theta_{\mathcal{M}}$. Notably, the 779247 representation which is not excluded by group theory arguments and could in principle show up among these constraints is explicitly absent. We shall come back to (a proof of) this fact in the next section.

The second equation in (3.51) implies that the singlet 1 and the vector 248 component of the embedding tensor cannot be switched on simultaneously. Absence of the vector $\theta_{\mathcal{M}}$ corresponds to the theories without gauging of the $\mathbb{R}^{+}$scaling symmetry. As these theories have been discussed in detail in [8], we shall in the following assume a non-vanishing vector $\theta_{\mathcal{M}}$ and thus a vanishing singlet component $\eta^{\mathcal{M N}} \Theta_{\mathcal{M N}}$ of the three-dimensional embedding tensor. 
Let us finally discuss the particular gaugings for which $\Theta_{\mathcal{M N}}=0$ and which are thus complementary to those constructed in [8]. In this case, the only non-trivial quadratic constraint on the remaining component $\theta_{K}$ comes from (3.49) and is given by

$$
\left(\left(\mathbb{P}_{\mathbf{1}}\right)_{\mathcal{M N}}{ }^{\mathcal{K} \mathcal{L}}+\left(\mathbb{P}_{\mathbf{3 8 7 5}}\right)_{\mathcal{M N}}{ }^{\mathcal{K} \mathcal{L}}\right) \theta_{\mathcal{K}} \theta_{\mathcal{L}} \stackrel{!}{\equiv} 0
$$

As in higher dimensions, this condition can thus be viewed as the "analogue of a pure spinor" (3.14) for the exceptional group $\mathrm{E}_{8(8)}$. We can use the same technology in order to find its general solution. As the calculation is somewhat more involved that for the higher-dimensional cases, we defer the details to appendix $\mathrm{B}$ and just present the solution here. Decomposing $\theta_{\mathcal{M}}$ under $\mathrm{E}_{7(7)} \times \mathbb{R}^{+}$into components $\left(\tilde{\eta}, \tilde{\eta}_{m}, \xi, \xi_{\alpha}, \eta_{m}, \eta\right)$ according to the decomposition 12

$$
248 \longrightarrow 1^{+2} \oplus 56^{+1} \oplus 1^{0} \oplus 133^{0} \oplus 56^{-1} \oplus 1^{-2},
$$

the general solution of (3.53) can be expressed in terms of the 58 parameters $\eta, \eta_{m}, \xi$ as

$$
\begin{aligned}
\xi_{\alpha} & =-\frac{6}{\eta}\left(t_{\alpha}\right)^{m n} \eta_{m} \eta_{n}, \\
\tilde{\eta}_{m} & =\frac{\xi}{\eta} \eta_{m}-\frac{24}{\eta^{2}}\left(t^{\alpha}\right)_{m}{ }^{n}\left(t_{\alpha}\right)^{p q} \eta_{n} \eta_{p} \eta_{q}, \\
\tilde{\eta} & =\frac{\xi^{2}}{\eta}-\frac{2}{\eta} \xi^{\alpha} \xi_{\alpha} .
\end{aligned}
$$

We note that the second term in $\tilde{\eta}$ is related to the quartic invariant $\left(t_{\alpha}\right)^{k l}\left(t^{\alpha}\right)^{m n} \eta_{k} \eta_{l} \eta_{m} \eta_{n}$ of $\mathrm{E}_{7(7)}$. Like for $\mathrm{E}_{7(7)}$ above, the explicit solution (3.55) in terms of 58 parameters shows that a vector subject to the bilinear condition (3.53) represents a very compact way to describe the non-linear conformal realization of $\mathrm{E}_{8(8)}$ given in 34 .

\subsection{Summary}

We have in this section explicitly constructed the gauge group generators of the gaugings of maximal supergravity that involve also a gauging of the on-shell scaling symmetry (1.1). In dimensions $3 \leq D \leq 6$, these generators are given by (2.18) with the respective values of $\zeta$ computed above in the various subsections. The possibility of a local scaling symmetry gives rise to another set of parameters $\theta_{M}$ within the embedding tensor that transform in the dual vector representation. We have worked out for all cases the quadratic constraints on the embedding tensor required for consistency. In particular, for those gaugings that are exclusively triggered by the new parameters $\theta_{M}$, we have furthermore given the explicit solution of these consistency constraints in all cases.

\footnotetext{
${ }^{12}$ Here, we use indices $m$ and $\alpha$ for the fundamental 56 and the adjoint 133 of $\mathrm{E}_{7(7)}$, respectively.
} 
While so far we have only derived the necessary algebraic consistency constraints, it remains to show that every solution to these constraints (e.g. to equations (3.36), (3.37) in $D=4$ dimensions) indeed gives rise to a consistent theory. In particular, it remains to determine the deformed field equations - as the theory no longer admits an action, the analysis must be performed on the level of the equations of motion. This will be the subject of the next section. We pick the example of the maximal $D=3$ supergravity, for which the algebraic structure is the most involved one, and show how the equations of motion must be modified under gauging in order to remain supersymmetric.

\section{Supersymmetry}

In this section we will take as an example the maximal three-dimensional theory and work out the full set of the deformed equations of motion. In particular, this will show that the quadratic constraints (3.49), (3.50) are sufficient for consistency of the theory, in other words, that every solution to these equations defines a consistent and maximally supersymmetric gauging in three dimensions. Upon dimensional reduction the algebraic structures which connect gauging and supersymmetry are embedded into the increasing symmetry algebras. The results of this section thus give some strong evidence that also the algebraic constraints we have derived in sections $3.1-3.3$ for the higher dimensions are sufficient for compatibility with supersymmetry.

As we have repeatedly mentioned, the resulting theory does not admit an action. The analysis must therefore be performed on the level of the equations of motion. After reviewing the three-dimensional theory we analyze the deformed supersymmetry algebra and in section 4.4 we derive the full set of the deformed equations of motion (to lowest order in the fermions).

\subsection{The three-dimensional theory}

We recall some basic notations of the maximal three-dimensional supergravity and its gaugings, see [35, 8] for details. Also we have collected in appendix A.1 our conventions for the exceptional group $\mathrm{E}_{8(8)}$.

The scalar fields in three dimensions are described by an $\mathrm{E}_{8(8)}$-valued matrix $\mathcal{V}^{\mathcal{M}} \underline{\mathcal{M}}$, with the two indices labeling the 248-dimensional adjoint representation and indicating the transformation properties

$$
\delta \mathcal{V}=\Lambda \mathcal{V}-\mathcal{V} h(x), \quad \Lambda \in \mathfrak{e}_{8(8)}, \quad h(x) \in \mathfrak{s o}(16)
$$

under global $\mathrm{E}_{8(8)}$ and local $\mathrm{SO}(16)$, respectively. In particular, it is customary to split the group matrix according to $\mathcal{V}^{\mathcal{M}} \underline{\mathcal{M}}=\left\{\mathcal{V}^{\mathcal{M}}{ }_{I J}, \mathcal{V}^{\mathcal{M}}{ }_{A}\right\}$, according to the decomposition of $\mathfrak{e}_{8(8)}$ into its compact subalgebra $\mathfrak{s o}(16)=\left\langle X^{[I J]}\right\rangle$ and 128 noncompact generators $\left\{Y^{A}\right\}$. Here $I, J, \ldots$ and $A, B, \ldots$, respectively, label the $\mathbf{1 6}$ and $\mathbf{1 2 8}_{s}$ representations 
of $\mathrm{SO}(16)$. Eventually we will also need indices $\dot{A}, \dot{B}, \ldots$ labelling the conjugate spinor representation $\mathbf{1 2 8}_{c}$. Naturally we will also encounter $\mathrm{SO}(16)$ gamma matrices $\Gamma_{A \dot{A}}^{I}$ in what follows. We will freely raise and lower $\mathrm{SO}(16)$ indices.

In this basis, the Cartan-Killing form $\eta_{\mathcal{M N}}$ of $\mathrm{E}_{8(8)}$ takes the form

$$
\eta_{\mathcal{M N}} \mathcal{V}^{\mathcal{M}}{ }_{I J} \mathcal{V}^{\mathcal{N}}{ }_{K L}=-2 \delta_{K L}^{I J}, \quad \eta_{\mathcal{M N}} \mathcal{V}^{\mathcal{M}}{ }_{A} \mathcal{V}^{\mathcal{N}}{ }_{B}=\delta_{A B}
$$

and the $\mathrm{E}_{8(8)}$ structure constants $f^{\mathcal{M N K}}$ can be expressed as

$$
\begin{aligned}
f^{\mathcal{M N K}} & =\mathcal{V}^{\mathcal{M}}{ }_{\underline{\mathcal{M}}} \mathcal{V}^{\mathcal{N}}{ }_{{ }_{\mathcal{N}}} \mathcal{V}^{\mathcal{K}}{ }_{\underline{\mathcal{K}}} f \frac{\mathcal{M N \mathcal { N }}}{} \\
& =-\mathcal{V}^{\mathcal{M}}{ }_{I J} \mathcal{V}^{\mathcal{N}}{ }_{K L} \mathcal{V}^{\mathcal{K}}{ }_{M N}\left(\delta^{I K} \delta^{L M} \delta^{N J}\right)-\frac{3}{4} \mathcal{V}^{\left[\mathcal{M}_{I J}\right.} \mathcal{V}^{\mathcal{N}}{ }_{A} \mathcal{V}^{\mathcal{K}]}{ }_{B}\left(\Gamma_{A B}^{I J}\right)
\end{aligned}
$$

The inverse matrix $\mathcal{V} \underline{\mathcal{M}}_{\mathcal{M}}$ is defined by 13

$$
\mathcal{V}^{K L}{ }_{\mathcal{M}} \mathcal{V}^{\mathcal{M}}{ }_{I J}=2 \delta_{K L}^{I J}, \quad \mathcal{V}_{\mathcal{M}}^{A} \mathcal{V}_{B}^{\mathcal{M}}=\delta_{B}^{A}
$$

The standard gaugings are defined in terms of the embedding tensor $\Theta_{\mathcal{M N}}$. The fermionic mass terms of the theory as well as the scalar potential can be expressed in terms of the $T$-tensor

$$
T_{\underline{\mathcal{M}} \mid \underline{\mathcal{N}}} \equiv \Theta_{\mathcal{M N}} \mathcal{V}^{\mathcal{M}} \underline{\underline{\mathcal{M}}} \mathcal{V}^{\mathcal{N}} \underline{\mathcal{N}}
$$

obtained by dressing the embedding tensor with the scalar matrix $\mathcal{V}^{\mathcal{M}} \underline{\mathcal{M}}$. Similarly, the crucial object in the description of the gaugings with local scaling symmetry will be the dressed new component $\theta_{\mathcal{M}}$ :

$$
T_{\underline{\mathcal{M}}} \equiv \theta_{\mathcal{M}} \mathcal{V}^{\mathcal{M}} \underline{\underline{\mathcal{M}}} .
$$

As $\Theta_{\mathcal{M N}}$ is restricted to live in the $\mathbf{1} \oplus \mathbf{3 8 7 5}$ representation of $\mathrm{E}_{8(8)}$, the same applies to the $T$-tensor. It can hence be expressed as

$$
\begin{aligned}
\Theta_{\mathcal{M N}}= & \mathcal{V} \underline{\mathcal{M}}_{\mathcal{M}} \mathcal{V} \underline{\mathcal{N}}_{\mathcal{N}} T_{\underline{\mathcal{M}} \mid \underline{\mathcal{N}}} \\
= & \frac{1}{4} \mathcal{V}_{\mathcal{M}}^{I J} \mathcal{V}_{\mathcal{N}}{ }^{K L}\left(\delta^{I[K} A_{1}^{L] J}-\delta^{J[K} A_{1}^{L] I}+\frac{1}{64} \Gamma_{\dot{A} \dot{B}}^{I J K L} A_{3}^{\dot{A} \dot{B}}\right) \\
& -\mathcal{V}_{(\mathcal{M}}{ }^{I J} \mathcal{V}_{\mathcal{N})}{ }^{A}\left(\Gamma_{A \dot{A}}^{[I} A_{2}^{J] \dot{A}}\right)+\mathcal{V}_{\mathcal{M}}{ }^{A} \mathcal{V}_{\mathcal{N}}{ }^{B}\left(\frac{1}{16} \Gamma_{A \dot{A}}^{I} \Gamma_{B \dot{B}}^{I} A_{3}^{\dot{A} \dot{B}}\right),
\end{aligned}
$$

in terms of three tensors $A_{1}, A_{2}$ and $A_{3}$ transforming in the $\mathbf{1 3 5}, \mathbf{1 9 2 0}_{c}$ and 1820 of $\mathrm{SO}(16)$, respectively, i.e. satisfying

$$
A_{1}^{I J}=A_{1}^{J I} \quad \Gamma_{A \dot{A}}^{I} A_{2}^{I \dot{A}}=0, \quad A_{3}^{\dot{A} \dot{B}}=\frac{1}{3072} \Gamma_{\dot{A} \dot{B}}^{I J K L} \Gamma_{\dot{C} \dot{D}}^{I J K L} A_{3}^{\dot{C} \dot{D}} .
$$

In the standard gauged theory (in absence of the $\mathbb{R}^{+}$-gauging), these terms describe the various fermionic mass term in the Lagrangian while the scalar potential is given by

$$
W(\phi)=\frac{1}{4} g^{2}\left(A_{2}^{I \dot{A}} A_{2}^{I \dot{A}}-2 A_{1}^{I J} A_{1}^{I J}\right) .
$$

\footnotetext{
${ }^{13}$ Note that these conventions gives rise to the relations $\mathcal{V}_{\mathcal{M}}{ }^{I J} \equiv \eta_{\mathcal{M N}} \mathcal{V}^{\mathcal{N} I J}=-\mathcal{V}^{I J} \mathcal{M}^{\text {and }} \mathcal{V}_{\mathcal{M}}{ }^{A} \equiv$ $\eta_{\mathcal{M N}} \mathcal{V}^{\mathcal{N} A}=\mathcal{V}^{A}{ }_{\mathcal{M}}$, cf. appendix A.1.
} 
Similarly, we now introduce tensors $B_{I J}$ and $B_{A}$ in order to parametrize the different $\mathrm{SO}(16)$ components of the new part (4.6) of the $T$-tensor

$$
\theta_{\mathcal{M}}=\mathcal{V}_{\mathcal{M}} \underline{\underline{\mathcal{M}}} T_{\underline{\mathcal{M}}}=\frac{1}{2} \mathcal{V}_{\mathcal{M}}^{I J} B_{I J}+\mathcal{V}_{\mathcal{M}}^{A} B_{A}
$$

With the currents

$$
\mathcal{V}^{-1}\left(\partial_{\mu}-g A_{\mu}^{\mathcal{M}} \widehat{\Theta}_{\mathcal{M N}} t^{\mathcal{N}}\right) \mathcal{V} \equiv \frac{1}{2} \mathcal{Q}_{\mu}^{I J} X^{I J}+\mathcal{P}_{\mu}^{A} Y^{A}
$$

we find among the various components of the $T$-tensor the differential relations

$$
\begin{aligned}
\mathcal{D}_{\mu} A_{1}^{I J} & =\Gamma_{A \dot{A}}^{(I} A_{2}^{J) \dot{A}} \mathcal{P}_{\mu}^{A}, \\
\mathcal{D}_{\mu} A_{2}^{I \dot{A}} & =\frac{1}{2}\left(\Gamma_{A \dot{A}}^{M} A_{1}^{I M}+\Gamma_{A \dot{B}}^{I} A_{3}^{\dot{A} \dot{B}}-\frac{1}{16}\left(\Gamma^{I} \Gamma^{J}\right)_{\dot{A} \dot{B}} \Gamma_{A \dot{C}}^{J} A_{3}^{\dot{C} \dot{B}}\right) \mathcal{P}_{\mu}^{A}, \\
\mathcal{D}_{\mu} B_{I J} & =\frac{1}{2} \Gamma_{A B}^{I J} B_{A} \mathcal{P}_{\mu}^{B}, \quad \mathcal{D}_{\mu} B_{A}=\frac{1}{4} \Gamma_{A B}^{I J} B_{I J} \mathcal{P}_{\mu}^{B},
\end{aligned}
$$

where $\mathcal{D}_{\mu}$ denotes the full $\mathrm{SO}(16)$-covariant derivative.

\subsection{Implications of the quadratic constraint}

In this section we will compute and collect a number of relations that can be derived from the quadratic constraints (3.49), (3.50) on the embedding tensor. The section is largely technical and since the algebraic calculations become quite involved we have made repeated use of the computer algebra system Cadabra [36] to organize and simplify the computation.

We have seen that the gauging of the theory is described in terms of the embedding tensor, which is parametrized by components $\Theta_{\mathcal{M N}}, \theta_{\mathcal{M}}$, subject to the relations (3.49), (3.50). The equations of motion of the theory on the other hand feature the dressed version of the embedding tensor defined in (4.5) and (4.6). In order to appreciate the consequences of the quadratic constraint, we will thus have to translate equations (3.49), (3.50) into relations between the scalar dependent tensors $A_{1,2,3}$ and $B$ from (4.7), (4.10).

Let us start from the simplest set of constraints (3.51). Its second equation translates into

$$
\eta^{\mathcal{M N}} \Theta_{\mathcal{M N}} B_{A}=0=\eta^{\mathcal{M N}} \Theta_{\mathcal{M N}} B_{I J}
$$

and as mentioned above, it is automatically solved if $\Theta_{\mathcal{M N}}$ transforms in the 3875 and has no singlet component. Plugging the explicit expansions (4.7), (4.10) into the remaining equations of (3.51) gives rise to the relations

$$
\begin{aligned}
B_{I J} B_{I J}-2 B_{A} B_{A} & =0, \\
A_{1}^{K[I} B^{J] K}+\Gamma_{A \dot{A}}^{[I} A_{2}^{J] \dot{A}} B_{A}-\frac{1}{128} \Gamma_{\dot{A} \dot{B}}^{I J K} A_{3}^{\dot{A} \dot{B}} B_{K L} & =0 \\
8 \Gamma_{A \dot{A}}^{I} A_{2}^{J \dot{A}} B_{I J}-\Gamma_{A \dot{A}}^{I} \Gamma_{B \dot{B}}^{I} A_{3}^{\dot{A} \dot{B}} B_{B} & =0 .
\end{aligned}
$$


We note that these constraints transform in the $\mathbf{1}, \mathbf{1 2 0}$, and $\mathbf{1 2 8}$ of $\mathrm{SO}(16)$, respectively. On the other hand, from evaluating (3.49) for $\mathcal{M}=[I K]$ and $\mathcal{N}=[J K]$ we obtain after subsequent symmetrisation and antisymmetrisation in $I, J$, two equations in the $\mathbf{1 3 5}$, and $\mathbf{1 2 0}$

$$
\begin{aligned}
8 B_{I K} B_{J K}+8 A_{1}^{K(I} B^{J) K}-4 \Gamma_{A \dot{A}}^{(I} A_{2}^{J) \dot{A}} B_{A}-\delta_{I J} B_{A} B_{A} & =0 \\
-6 A_{1}^{K[I} B^{J] K}-3 \Gamma_{A \dot{A}}^{[I} A_{2}^{J] \dot{A}} B_{A}+\frac{1}{64} \Gamma_{\dot{A} \dot{B}}^{I J K L} A_{3}^{\dot{A} \dot{B}} B_{K L} & =0
\end{aligned}
$$

respectively. Instead, choosing in (3.49) $\mathcal{M}=[I J]$ and $\mathcal{N}=A$ and contracting the equation with $\Gamma_{A \dot{A}}^{J}$ leads to

$$
\begin{aligned}
0= & 7 \Gamma_{A \dot{A}}^{J} B_{I J} B_{A}-\frac{7}{16} \Gamma_{A \dot{A}}^{I} \Gamma_{A B}^{J K} B_{J K} B_{B}+\frac{7}{2} \Gamma_{A \dot{A}}^{J} A_{1}^{I J} B_{A}-A_{2}^{J \dot{A}} B_{I J}+\frac{3}{2} \Gamma_{A \dot{B}}^{I} A_{3}^{\dot{A} \dot{B}} B_{A} \\
& -\frac{1}{2}\left(\Gamma^{I} \Gamma^{K}\right)_{\dot{A} \dot{B}} A_{2}^{J \dot{B}} B_{J K}-\frac{5}{4} \Gamma_{\dot{A} \dot{B}}^{J K} A_{2}^{I \dot{B}} B_{J K}-\frac{3}{16}\left(\Gamma^{I} \Gamma^{J}\right)_{\dot{A} \dot{B}} \Gamma_{B \dot{C}}^{J} A_{3}^{\dot{B} \dot{C}} B_{B}
\end{aligned}
$$

Upon interchanging $\mathcal{M}$ and $\mathcal{N}$ in (3.49), the same contraction yields

$$
\begin{aligned}
0= & 7 \Gamma_{A \dot{A}}^{J} B_{I J} B_{A}-\frac{7}{16} \Gamma_{A \dot{A}}^{I} \Gamma_{A B}^{J K} B_{J K} B_{B}-6 A_{2}^{J \dot{A}} B_{I J}+2 \Gamma_{A \dot{B}}^{I} A_{3}^{\dot{A} \dot{B}} B_{A} \\
& +\frac{1}{2}\left(\Gamma^{I} \Gamma^{K}\right)_{\dot{A} \dot{B}} A_{2}^{J \dot{B}} B_{J K}-\frac{1}{2} \Gamma_{\dot{A} \dot{B}}^{J K} A_{2}^{I \dot{B}} B_{J K}-\frac{1}{32}\left(\Gamma^{I} \Gamma^{J}\right)_{\dot{A} \dot{B}} \Gamma_{B \dot{C}}^{J} A_{3}^{\dot{B} \dot{C}} B_{B} .
\end{aligned}
$$

Under SO(16) the two equations (4.16) and (4.17) transform in the $\mathbf{1 2 8}_{s} \oplus \mathbf{1 9 2 0}_{c}$ and it is straightforward to verify that the two parts in the $\mathbf{1 2 8}_{s}$ (obtained by further contraction with $\Gamma_{B \dot{A}}^{I}$ ) are proportional to the last equation of (4.14).

Finally, we evaluate part of the quadratic constraint (3.50). Choosing $\mathcal{M}=[J K]$, $\mathcal{N}=[I M], \mathcal{P}=[K M]$ and symmetrizing in $(I J)$ leads to the relation

$$
\begin{aligned}
0= & A_{1}^{I K} A_{1}^{J K}-\frac{1}{2} A_{2}^{I \dot{A}} A_{2}^{J \dot{A}}+A_{1}^{K(I} B^{J) K}-\frac{1}{4} \Gamma_{A \dot{A}}^{(I} A_{2}^{J) \dot{A}} B_{A} \\
& -\frac{1}{16} \delta^{I J}\left(A_{1}^{K L} A_{1}^{K L}-\frac{1}{2} A_{2}^{K \dot{A}} A_{2}^{K \dot{A}}\right)
\end{aligned}
$$

in the 135 of $\mathrm{SO}(16)$. Choosing in (3.50) $\mathcal{M}=A, \mathcal{N}=[I M], \mathcal{P}=[K M]$ and contracting with $\Gamma_{A \dot{A}}^{K}$ we obtain

$$
\begin{aligned}
\frac{1}{64} \Gamma_{\dot{C} \dot{D}}^{I J K L} \Gamma_{\dot{A} \dot{B}}^{K L} A_{2}^{J \dot{B}} A_{3}^{\dot{C} \dot{D}}= & -32 A_{1}^{I J} A_{2}^{J \dot{A}}+2\left(\Gamma^{I} \Gamma^{J}\right)_{\dot{A} \dot{B}} A_{1}^{J K} A_{2}^{J \dot{B}}+10 A_{2}^{I \dot{B}} A_{3}^{\dot{A} \dot{B}} \\
& -\left(\Gamma^{I} \Gamma^{J}\right)_{\dot{A} \dot{B}} A_{2}^{J \dot{C}} A_{3}^{\dot{B} \dot{C}}+20 \Gamma_{A \dot{A}}^{J} A_{1}^{I J} B_{A}+2 A_{2}^{J \dot{A}} B_{I J} \\
& -\frac{7}{2} \Gamma_{\dot{A} \dot{B}}^{J K} A_{2}^{I \dot{B}} B_{J K}+\left(\Gamma^{I} \Gamma^{K}\right)_{\dot{A} \dot{B}} A_{2}^{J \dot{B}} B_{J K}
\end{aligned}
$$

Choosing instead $\mathcal{M}=[I M], \mathcal{N}=[K M], \mathcal{P}=A$ before contracting with $\Gamma_{A \dot{A}}^{K}$, we obtain

$$
\begin{aligned}
\frac{1}{64} \Gamma_{\dot{C} \dot{D}}^{I J K} \Gamma_{\dot{A} \dot{B}}^{K L} A_{2}^{J \dot{B}} A_{3}^{\dot{C} \dot{D}}= & 64 A_{1}^{I J} A_{2}^{J \dot{A}}-4\left(\Gamma^{I} \Gamma^{J}\right)_{\dot{A} \dot{B}} A_{1}^{J K} A_{2}^{J \dot{B}}-22 A_{2}^{I \dot{B}} A_{3}^{\dot{A} \dot{B}} \\
& +\left(\Gamma^{I} \Gamma^{J}\right)_{\dot{A} \dot{B}} A_{2}^{J \dot{C}} A_{3}^{\dot{B} \dot{C}}+\Gamma_{A \dot{A}}^{J} A_{1}^{I J} B_{A}+64 A_{2}^{J \dot{A}} B_{I J} \\
& -2\left(\Gamma^{I} \Gamma^{K}\right)_{\dot{A} \dot{B}} A_{2}^{J \dot{B}} B_{J K}-11 \Gamma_{A \dot{B}}^{I} A_{3}^{\dot{A} \dot{B}} B_{A} \\
& -\frac{1}{16}\left(\Gamma^{I} \Gamma^{J}\right)_{\dot{A} \dot{B}} \Gamma_{A \dot{C}}^{J} A_{3}^{\dot{B} \dot{C}} B_{A}
\end{aligned}
$$


Again these two equations transform in the $\mathbf{1 2 8}_{s} \oplus \mathbf{1 9 2 0}_{c}$ and one verifies that both $\mathbf{1 2 8}$ s parts reduce to the last equation of (4.14). We note that in absence of the vector $\theta_{\mathcal{M}}$, (i.e. for $B_{I J}=0=B_{A}$ ) all these equations consistently reduce to equations (4.17) and (4.19) of [8]. Together, we have thus shown that the lowest $\mathrm{SO}(16)$ representations appearing in the quadratic constraint are given by

$$
\mathcal{R}_{\text {quad }}=1 \oplus 3 \cdot \mathbf{1 2 0} \oplus 2 \cdot \mathbf{1 2 8}_{s} \oplus 3 \cdot \mathbf{1 3 5} \oplus 4 \cdot \mathbf{1 9 2 0}_{c} \oplus \ldots
$$

in agreement with the corresponding decomposition of (3.52). In particular, the fact that within all the above equations there are only two independent constraints in the $\mathbf{1 2 8}_{s}$ finally proves that there is no $\mathrm{E}_{8(8)}$ representation $\mathbf{7 7 9 2 4 7}$ in the quadratic constraint (3.52). Its presence would have excluded all solutions to the quadratic constraint with both $\Theta_{\mathcal{M N}}$ and $\theta_{\mathcal{M}}$ non-vanishing.

In order to study supersymmetry of the equations of motion in the next section, we will need the following particular linear combinations of the above constraints in the the $128_{s} \oplus 1920_{c}$ representation

$$
\begin{aligned}
0= & 3 A_{1}^{I J} A_{2}^{J \dot{A}}-A_{2}^{I \dot{B}} A_{3}^{\dot{A} \dot{B}}+3 A_{2}^{J \dot{A}} B_{I J}-\frac{1}{4} A_{2}^{I \dot{B}} B_{J K} \Gamma_{\dot{A} \dot{B}}^{J K}+\frac{3}{4} A_{1}^{I J} B_{A} \Gamma_{A \dot{A}}^{J} \\
& -\frac{1}{4} A_{3}^{\dot{A} \dot{B}} B_{A} \Gamma_{A \dot{B}}^{I}+B_{A} B_{I J} \Gamma_{A \dot{A}}^{J}-\frac{3}{16} A_{1}^{J K} A_{2}^{K \dot{B}}\left(\Gamma^{I} \Gamma^{J}\right)_{\dot{A} \dot{B}}+\frac{1}{16} A_{2}^{J \dot{C}} A_{3}^{\dot{B} \dot{C}}\left(\Gamma^{I} \Gamma^{J}\right)_{\dot{A} \dot{B}} \\
& -\frac{1}{4} A_{2}^{K \dot{B}} B_{J K}\left(\Gamma^{I} \Gamma^{J}\right)_{\dot{A} \dot{B}}+\frac{1}{64} A_{3}^{\dot{B} \dot{C}} B_{A}\left(\Gamma^{I} \Gamma^{J}\right)_{\dot{A} \dot{B}} \Gamma_{A \dot{C}}^{J}-\frac{1}{16} B_{A} B_{J K}\left(\Gamma^{I} \Gamma^{J K}\right)_{\dot{A} A} .
\end{aligned}
$$

Again, in absence of $B_{I J}$ and $B_{A}$ this equation consistently reduces to the constraint derived in [8], section 4.4 .

\subsection{Supersymmetry algebra}

We will now study the effect of the gauging on the three-dimensional supersymmetry algebra. This will allow us to derive the deformed supersymmetry transformation rules which we will subsequently use to determine the full set of deformed field equations. For the standard gaugings (in absence of the vector $\theta_{\mathcal{M}}$ ), the supersymmetry algebra in three dimensions has recently been computed for all $p$-forms [31].

For the bosonic fields $e_{\mu}{ }^{\alpha}, \mathcal{V}$ and $A_{\mu}^{\mathcal{M}}$, the supersymmetry transformation rules are given by

$$
\begin{aligned}
\delta e_{\mu}{ }^{\alpha} & =\mathrm{i} \bar{\epsilon}^{I} \gamma^{\alpha} \psi_{\mu}{ }^{I}, \quad \mathcal{V}^{-1} \delta \mathcal{V}=\Gamma_{A \dot{A}}^{I} \bar{\chi}^{\dot{A}} \epsilon^{I} Y^{A} \\
\delta A_{\mu}{ }^{\mathcal{M}} & =2 \mathcal{V}^{\mathcal{M}}{ }_{I J} \bar{\epsilon}^{I} \psi_{\mu}{ }^{J}-\mathrm{i} \Gamma_{A \dot{A}}^{I} \mathcal{V}^{\mathcal{M}}{ }_{A} \bar{\epsilon}^{I} \gamma_{\mu} \chi^{\dot{A}}
\end{aligned}
$$

and do not change upon gauging, 14 The fermionic fields appearing in these transformations are 16 gravitinos $\psi_{\mu}^{I}$ and 128 spin-1/2 fermions $\chi^{\dot{A}}$ transforming under $\mathrm{SO}(16)$.

\footnotetext{
${ }^{14}$ Our space-time conventions are a signature $(+--)$ for the three-dimensional metric $g_{\mu \nu}$, and $e \gamma^{\mu \nu \rho}=-\mathrm{i} \varepsilon^{\mu \nu \rho}$ for the $\operatorname{SO}(1,2) \gamma$-matrices.
} 
In the presence of a gauging their supersymmetry variations are given (up to higher order fermionic contributions) by

$$
\begin{aligned}
\delta_{\epsilon} \psi_{\mu}^{I} & =\mathcal{D}_{\mu} \epsilon^{I}+i g\left(A_{1}^{I J}+\beta_{1} B_{I J}\right) \gamma_{\mu} \epsilon^{J} \\
\delta_{\epsilon} \chi^{\dot{A}} & =\frac{i}{2} \gamma^{\mu} \epsilon^{I} \Gamma_{A \dot{A}}^{I} \mathcal{P}_{\mu}^{A}+g\left(A_{2}^{I \dot{A}}+\beta_{2} \Gamma_{A \dot{A}}^{I} B_{A}\right) \epsilon^{I}
\end{aligned}
$$

with the tensors $A_{1}, A_{2}$ and $B$ from (4.7), (4.10) above and some constants $\beta_{1,2}$. The covariant derivative is explicitly given by

$$
\mathcal{D}_{\mu} \epsilon^{I}=\left(\partial_{\mu}+\frac{1}{4} \widehat{\omega}_{\mu}^{a b} \gamma_{a b}-\frac{1}{2} \mathcal{A}_{\mu}\right) \epsilon^{I}+\mathcal{Q}_{\mu}^{I J} \epsilon^{J}
$$

The effect of a gauging with non-vanishing vector $\theta_{\mathcal{M}}$ in these transformations is furthermore reflected by the terms in $B_{I J}$ and $B_{A}$ which are entirely determined by their index structure up to the global factors $\beta_{1,2}$. The latter are fixed by demanding closure of the supersymmetry algebra into diffeomorphisms, Lorentz transformations, $\mathrm{SO}(16)$ transformations and gauge transformations:

$$
\left[\delta_{\epsilon_{1}}, \delta_{\epsilon_{2}}\right]=\delta_{\xi}+\delta_{\omega}+\delta_{h}+\delta_{\Lambda}
$$

Setting $\beta_{1}=-1, \beta_{2}=\frac{1}{4}$, one can verify that the supersymmetry transformations (4.23), (4.24) close on the vielbein $e_{\mu}{ }^{\alpha}$ and on the scalar fields $\mathcal{V}$ into the algebra (4.26) with diffeomorphism and gauge parameter given by

$$
\begin{aligned}
\xi^{\mu} & =-\mathrm{i} \bar{\epsilon}_{[1}^{I} \gamma^{\mu} \epsilon_{2]}^{I}, \\
\Lambda^{\mathcal{M}} & =-\xi^{\rho} A_{\rho}^{\mathcal{M}}-2 \mathcal{V}^{\mathcal{M}}{ }_{I J} \bar{\epsilon}_{[1}^{I} \epsilon_{2]}^{J} .
\end{aligned}
$$

On the vector fields, the commutator of two supersymmetry transformations yields (again up to higher order fermionic terms)

$$
\begin{aligned}
{\left[\delta_{\epsilon_{1}}, \delta_{\epsilon_{2}}\right] A_{\mu}^{\mathcal{M}}=} & \left(\delta_{\xi}+\delta_{\Lambda}\right) A_{\mu}^{\mathcal{M}}-\frac{4}{7} g\left(Z^{\mathcal{M}}{ }_{\mathcal{P} \mathcal{Q}} \mathcal{V}^{\mathcal{P}}{ }_{I K} \mathcal{V}^{\mathcal{Q}}{ }_{J K}-\theta^{\mathcal{M}} \delta_{I J}\right) \xi_{\mu}^{I J} \\
& -\xi^{\nu}\left(\mathcal{F}_{\mu \nu}^{\mathcal{M}}+e \varepsilon_{\mu \nu \rho} \mathcal{V}^{\mathcal{M}}{ }_{A} \mathcal{P}^{\rho A}\right)
\end{aligned}
$$

with $\xi_{\mu}^{I J}=-\mathrm{i} \bar{\epsilon}_{[1}^{I} \gamma^{\mu} \epsilon_{2]}^{J}$ and the non-abelian field strength

$$
\mathcal{F}_{\mu \nu}^{\mathcal{M}}=2 \partial_{[\mu} A_{\nu]}^{\mathcal{M}}+g X_{[\mathcal{N} \mathcal{P}]}^{\mathcal{M}} A_{\mu}^{\mathcal{N}} A_{\nu}^{\mathcal{P}}
$$

In order to arrive at this result, one needs the explicit expression of the intertwining tensor $Z^{\mathcal{M}}{ }_{\mathcal{P} \mathcal{Q}}$ which may be obtained after some calculation by plugging (4.7), (4.10) into (3.47):

$$
\begin{aligned}
Z^{\mathcal{M}}{ }_{\mathcal{P Q}} \mathcal{V}^{\mathcal{P}}{ }_{I K} \mathcal{V}^{\mathcal{Q}}{ }_{J K}= & -\frac{7}{2}\left(\mathcal{V}^{\mathcal{M}}{ }_{I K} A_{1}^{K J}+\mathcal{V}^{\mathcal{M}}{ }_{J K} A_{1}^{K I}\right)+\frac{7}{2} \mathcal{V}^{\mathcal{M}}{ }_{A} \Gamma_{A \dot{A}}^{(I} A_{2}^{J) \dot{A}} \\
& +7 \mathcal{V}^{\mathcal{M}}{ }_{K(I} B_{J) K}-\frac{1}{2}\left(\mathcal{V}^{\mathcal{M}}{ }_{K L} B_{K L}-\frac{15}{4} \mathcal{V}^{\mathcal{M}}{ }_{A} B_{A}\right) \delta_{I J}
\end{aligned}
$$


A priori, the result (4.28) differs by its last two terms from the expected supersymmetry algebra (4.26). The last term is precisely the duality equation between scalars and vector fields in three dimensions and signifies the fact that the supersymmetry algebra closes only modulo the equations of motion [31]. In order to understand the second term in (4.28) we recall that in the gauged theory the vector fields always appear contracted as $\widehat{\Theta}_{\mathcal{M N}} A_{\mu}^{\mathcal{M}}$ or $\theta_{\mathcal{M}} A_{\mu}^{\mathcal{M}}$. Under this contraction, the second term in (4.28) consistently vanishes as a result of the quadratic constraints (2.27) and (3.51) 15

We have thereby established the full set of deformed supersymmetry transformation rules for the general gauged theory in three dimensions.

\subsection{Equations of motion}

We have now all the ingredients to derive the full deformed theory. As the gaugings with local scaling symmetry do no longer admit an action, one must consider the deformation directly on the level of the equations of motion. The general gauging is parametrized by an embedding tensor with components $\Theta_{\mathcal{M N}}, \theta_{\mathcal{M}}$ which defines covariant derivatives according to (2.15), (2.18). For non-vanishing $\theta_{\mathcal{M}}$ the gauge group also includes the generator of the scaling symmetry (1.1). The embedding tensor defines the scalar field dependent tensors $A_{1,2,3}$ and $B$ which show up in the modified supersymmetry transformation rules (4.23), (4.23) derived in the last subsection.

In the computation of the supersymmetry algebra (4.28), we have already met the first dynamical equations

$$
\begin{aligned}
\widehat{\Theta}_{\mathcal{M N}}\left(\mathcal{F}_{\mu \nu}{ }^{\mathcal{M}}+e \varepsilon_{\mu \nu \rho} \mathcal{V}_{A}^{\mathcal{M}} \mathcal{P}^{\rho A}\right) & =0, \\
\theta_{\mathcal{M}}\left(\mathcal{F}_{\mu \nu}{ }^{\mathcal{M}}+e \varepsilon_{\mu \nu \rho} \mathcal{V}^{\mathcal{M}}{ }_{A} \mathcal{P}^{\rho A}\right) & =0 .
\end{aligned}
$$

Note that this first order duality equation between vector and scalar fields is only imposed under projection with $\widehat{\Theta}_{\mathcal{M N}}$ and $\theta_{\mathcal{M}}$, respectively. This implies that not the full set of bosonic field equations but only a projection thereof can be retrieved from integrability of this equation. In particular, all contributions from a possible scalar potential will be invisible in the second order scalar field equations obtained from (4.31).

In order to find the full set of field equations, we start from the equations of motion of the gravitino of the ungauged theory [35]

$$
i \gamma^{\rho \mu \nu} D_{\mu} \psi_{\nu}^{I}-\frac{1}{2} \gamma^{\nu} \gamma^{\rho} \chi^{\dot{A}} \Gamma_{A \dot{A}}^{I} P_{\nu}^{A}=0
$$

Upon gauging, derivatives are covariantized, i.e. $D_{\mu} \rightarrow \mathcal{D}_{\mu}, P_{\mu}^{A} \rightarrow \mathcal{P}_{\mu}^{A}$. Moreover, in absence of a $\theta_{\mathcal{M}}$ the right-hand side of this equation is modified by terms proportional to the tensors $A_{1}$ and $A_{2}$ from (4.7) [8]. It is thus natural to assume that for the full

\footnotetext{
${ }^{15} \mathrm{As}$ in 31 one may alternatively absorb this term into additional gauge transformations related to the further introduction of two-form tensor fields.
} 
gauging the r.h.s. also receives corrections proportional to the tensors $B_{I J}$ and $B_{A}$. Up to factors $\alpha_{1,2}$, these are entirely determined by their $\mathrm{SO}(16)$ structure:

$$
\begin{aligned}
i \gamma^{\rho \mu \nu} \mathcal{D}_{\mu} \psi_{\nu}^{I}-\frac{1}{2} \gamma^{\nu} \gamma^{\rho} \chi^{\dot{A}} \Gamma_{A \dot{A}}^{I} \mathcal{P}_{\nu}^{A}= & -g\left(A_{1}^{I K}+\alpha_{1} B_{I K}\right) \gamma^{\rho \nu} \psi_{\nu}^{K} \\
& +i g\left(A_{2}^{I \dot{A}}+\alpha_{2} \Gamma_{A \dot{A}}^{I} B_{A}\right) \gamma^{\rho} \chi^{\dot{A}}
\end{aligned}
$$

In order to verify consistency and to determine the factors $\alpha_{1,2}$ we compute the transformation of this equation under supersymmetry. We will in this calculation neglect cubic terms in the fermions, i.e. only consider variation of the fermionic fields in (4.33).

The first term gives rise to a contribution involving the commutator of two covariant derivatives (4.25) which can be simplified using (2.11) to

$$
\begin{aligned}
i \gamma^{\rho \mu \nu} \mathcal{D}_{\mu} \mathcal{D}_{\nu} \epsilon^{I} & =\frac{i}{2} \gamma^{\rho \mu \nu}\left(\mathcal{Q}_{\mu \nu}^{I J} \epsilon^{J}-\frac{1}{2} \mathcal{F}_{\mu \nu} \epsilon^{I}+\frac{1}{4} \widehat{\mathcal{R}}_{\mu \nu}{ }^{a b} \gamma_{a b} \epsilon^{I}\right) \\
& =\frac{i}{2} \gamma \mathcal{Q}_{\mu \nu}^{I J} \epsilon^{J}+\frac{i}{2}\left(\left(\widehat{\mathcal{R}}^{(\rho \mu)}-\frac{1}{2} g^{\rho \mu} \widehat{\mathcal{R}}\right) \gamma_{\mu}-\frac{1}{2} \mathcal{F}_{\mu \nu} \gamma^{\rho \mu \nu}-\frac{1}{2} \mathcal{F}^{\rho \mu} \gamma_{\mu}\right) \epsilon^{I},
\end{aligned}
$$

with the abelian field strength $\mathcal{F}_{\mu \nu}=g \theta_{\mathcal{M}} \mathcal{F}_{\mu \nu}^{\mathcal{M}}$ and

$$
\mathcal{Q}_{\mu \nu}^{I J} \equiv 2 \partial_{[\mu} \mathcal{Q}_{\nu]}^{I J}+2 \mathcal{Q}_{\mu}^{K[I} \mathcal{Q}_{\nu}^{J] K}=-\frac{1}{2} \Gamma_{A B}^{I J} \mathcal{P}_{\mu}^{A} \mathcal{P}_{\nu}^{B}-g \mathcal{F}_{\mu \nu}^{\mathcal{M}} \widehat{\Theta}_{\mathcal{M N}} \mathcal{V}^{\mathcal{N}}{ }_{I J}
$$

obtained from integrability of (4.11). Likewise, variation of the second term on the l.h.s. of (4.33) creates terms bilinear in $\mathcal{P}_{\mu}^{A}$ which after some calculation simplify to

$$
\frac{i}{4} \gamma^{\rho \mu \nu} \Gamma_{A B}^{I J} \mathcal{P}_{\mu}^{A} \mathcal{P}_{\nu}^{B} \epsilon^{J}-\frac{1}{2} i\left(\mathcal{P}^{\rho A} \mathcal{P}^{\mu A}-\frac{1}{2} g^{\rho \mu} \mathcal{P}^{\nu A} \mathcal{P}_{\nu}^{A}\right) \gamma_{\mu} \epsilon^{I}
$$

The total variation of the l.h.s. of (4.33) is thus given by the sum of (4.34) and (4.36) together with the order $g$ contributions from (4.24). Altogether we obtain

$$
\begin{aligned}
\delta_{\epsilon}(\text { l.h.s. })= & \frac{1}{2} i\left(\widehat{\mathcal{R}}^{(\rho \mu)}-\frac{1}{2} g^{\rho \mu} \widehat{\mathcal{R}}-\mathcal{P}^{\rho A} \mathcal{P}^{\mu A}+\frac{1}{2} g^{\rho \mu} \mathcal{P}^{\nu A} \mathcal{P}_{\nu}^{A}\right) \gamma_{\mu} \epsilon^{I} \\
& -\frac{1}{2} i g \gamma^{\rho \mu \nu} \mathcal{F}_{\mu \nu}^{\mathcal{M}} \widehat{\Theta}_{\mathcal{M N}} \mathcal{V}^{\mathcal{N}}{ }_{I J} \epsilon^{J}-\frac{1}{4} i \gamma^{\rho \mu \nu} \mathcal{F}_{\mu \nu} \epsilon^{I}-\frac{1}{4} i \mathcal{F}^{\rho \mu} \gamma_{\mu} \epsilon^{I} \\
& -g \gamma^{\rho \mu \nu} \mathcal{D}_{\mu}\left(\left(A_{1}^{I J}-B_{I J}\right) \gamma_{\nu} \epsilon^{J}\right)-\frac{1}{2} g \gamma^{\nu} \gamma^{\rho} \Gamma_{A \dot{A}}^{I} \mathcal{P}_{\nu}^{A}\left(A_{2}^{J \dot{A}}+\frac{1}{4} \Gamma_{B \dot{A}}^{J} B_{B}\right) \epsilon^{J}
\end{aligned}
$$

Using the duality equations (4.31) to replace the various field strengths and the differential relations (4.12), this variation reduces to

$$
\begin{aligned}
\delta_{\epsilon}(\text { l.h.s. })= & \frac{1}{2} i\left(\widehat{\mathcal{R}}^{(\rho \mu)}-\frac{1}{2} g^{\rho \mu} \widehat{\mathcal{R}}-\mathcal{P}^{\rho A} \mathcal{P}^{\mu A}+\frac{1}{2} g^{\rho \mu} \mathcal{P}^{\nu A} \mathcal{P}_{\nu}^{A}\right) \gamma_{\mu} \epsilon^{I} \\
& +g \Gamma_{A \dot{A}}^{[I} A_{2}^{J]} \mathcal{P}^{\rho A} \epsilon^{J}-\frac{1}{4} g \Gamma_{A B}^{I J} B_{B} \mathcal{P}^{\rho A} \epsilon^{J}+\frac{1}{2} g B_{A} \mathcal{P}^{\rho A} \epsilon^{I}+\frac{1}{4} g \gamma^{\rho \mu} B_{A} \mathcal{P}_{\mu}^{A} \epsilon^{I} \\
& -g \gamma^{\rho \mu}\left(\Gamma_{A \dot{A}}^{(I} A_{2}^{J) \dot{A}}+\frac{1}{2} \Gamma_{A B}^{I J} B_{B}\right) \mathcal{P}_{\mu}^{A} \epsilon^{J}-g \gamma^{\rho \mu \nu}\left(A_{1}^{I J}-B_{I J}\right) \gamma_{\nu} \mathcal{D}_{\mu} \epsilon^{J} \\
& +\frac{1}{2} g \gamma^{\rho \nu} \Gamma_{A \dot{A}}^{I}\left(A_{2}^{J \dot{A}}+\frac{1}{4} \Gamma_{B \dot{A}}^{J} B_{B}\right) \mathcal{P}_{\nu}^{A} \epsilon^{J}-\frac{1}{2} g \Gamma_{A \dot{A}}^{I}\left(A_{2}^{J \dot{A}}+\frac{1}{4} \Gamma_{B \dot{A}}^{J} B_{B}\right) \mathcal{P}^{\rho A} \epsilon^{J},
\end{aligned}
$$


where we have also made use of the relation $\mathcal{V}^{\mathcal{M}} \widehat{\Theta}_{\mathcal{M N}} \mathcal{V}^{\mathcal{N}}{ }_{I J}=\Gamma_{A \dot{A}}^{[I} A_{2}^{J] \dot{A}}+\frac{1}{4} \Gamma_{A B}^{I J} B_{B}$, obtained from (2.18) with (4.7), (4.10). Collecting terms, we finally arrive at

$$
\begin{aligned}
\delta_{\epsilon}(\text { l.h.s. })= & \frac{1}{2} i\left(\widehat{\mathcal{R}}^{(\rho \mu)}-\frac{1}{2} g^{\rho \mu} \widehat{\mathcal{R}}-\mathcal{P}^{\rho A} \mathcal{P}^{\mu A}+\frac{1}{2} g^{\rho \mu} \mathcal{P}^{\nu A} \mathcal{P}_{\nu}^{A}\right) \gamma_{\mu} \epsilon^{I} \\
& -\frac{1}{8} g\left(4 \Gamma_{A \dot{A}}^{J} A_{2}^{I \dot{A}}+3 \Gamma_{A B}^{I J} B_{B}-3 \delta^{I J} B_{A}\right)\left(\mathcal{P}^{\rho A}+\gamma^{\rho \mu} \mathcal{P}_{\mu}^{A}\right) \epsilon^{J} \\
& -g\left(A_{1}^{I J}-B_{I J}\right) \gamma^{\rho \nu} \mathcal{D}_{\nu} \epsilon^{J} .
\end{aligned}
$$

While the first term will be part of the Einstein equations, the remaining terms cannot be part of any bosonic equations of motion and must therefore be cancelled by the variation of the r.h.s. of (4.33). As this variation is given by

$$
\begin{aligned}
\left.\delta_{\epsilon} \text { (r.h.s. }\right)= & -g\left(A_{1}^{I J}+\alpha_{1} B_{I J}\right) \gamma^{\rho \nu} \mathcal{D}_{\nu} \epsilon^{J} \\
& -\frac{1}{2} g \Gamma_{A \dot{A}}^{J}\left(A_{2}^{I \dot{A}}+\alpha_{2} \Gamma_{B \dot{A}}^{I} B_{B}\right) \gamma^{\rho} \gamma^{\mu} \mathcal{P}_{\mu}^{A} \epsilon^{J}+\mathcal{O}\left(g^{2}\right),
\end{aligned}
$$

one observes immediately that with $\alpha_{1}=-1, \alpha_{2}=-\frac{3}{4}$, all terms in order $g$ cancel against (4.37). It remains to study the order $g^{2}$ terms in (4.38). Note that by now we have fixed all free parameters, i.e. the remaining terms pose a non-trivial consistency check on the supersymmetry of the equations of motion. Applying the order $g$ variation on the r.h.s. of (4.33), we obtain

$$
\begin{aligned}
\delta_{\epsilon}^{\left(g^{2}\right)}(\text { r.h.s. })= & -2 i g^{2}\left(A_{1}^{I K}+\alpha_{1} B_{I K}\right)\left(A_{1}^{K J}-B_{K J}\right) \gamma^{\rho} \epsilon^{J} \\
& +i g^{2}\left(A_{2}^{I \dot{A}}+\alpha_{2} \Gamma_{A \dot{A}}^{I} B_{A}\right)\left(A_{2}^{J \dot{A}}+\frac{1}{4} \Gamma_{B \dot{A}}^{J} B_{B}\right) \gamma^{\rho} \epsilon^{J} .
\end{aligned}
$$

The result can be simplified upon expanding the products and using the bilinear relations between the tensors $A_{1,2,3}, B$, derived in section 4.2. We first observe that the combination $2 A_{1} A_{1}-A_{2} A_{2}$ can be replaced using (4.18). Furthermore, by virtue of (4.14) and (4.15) we can eliminate all the $\Gamma^{I} B_{A} A_{2}$ terms and obtain altogether

$$
\begin{aligned}
\delta_{\epsilon}^{\left(g^{2}\right)}(\text { r.h.s. })= & -i g^{2}\left(\left(2\left(\alpha_{1}-\alpha_{2}\right)+\frac{1}{2}\right) B_{I K} B_{J K}+\left(2 \alpha_{1}-3 \alpha_{2}-\frac{1}{4}\right) B_{I K} A_{1}^{J K}\right. \\
& \left.+\left(\alpha_{2}+\frac{3}{4}\right) B_{J K} A_{1}^{I K}-\frac{1}{16} \delta^{I J}\left(2 A_{1}^{K L} A_{1}^{K L}-A_{2}^{K \dot{A}} A_{2}^{K \dot{A}}-B_{A} B_{A}\right)\right) \gamma^{\rho} \epsilon^{J} .
\end{aligned}
$$

Remarkably, with the choice $\alpha_{1}=-1, \alpha_{2}=-\frac{3}{4}$ imposed earlier, the first three terms in this variation vanish and the result is again proportional to $\delta^{I J}$ and can thus be absorbed into the Einstein equations, as required for consistency. Combining (4.40) with (4.37) and (4.38) we thus finally obtain the modified Einstein equation

$$
\widehat{\mathcal{R}}_{(\mu \nu)}-\frac{1}{2} g_{\mu \nu} \widehat{\mathcal{R}}=\mathcal{P}_{\mu}^{A} \mathcal{P}_{\nu}^{A}-\frac{1}{2} g_{\mu \nu} \mathcal{P}^{\rho A} \mathcal{P}_{\rho}^{A}-\frac{1}{2} g_{\mu \nu} W(\phi)
$$

with

$$
W(\phi) \equiv \frac{1}{4} g^{2}\left(A_{2}^{I \dot{A}} A_{2}^{I \dot{A}}+B_{A} B_{A}-2 A_{1}^{I J} A_{1}^{I J}\right)
$$


playing the role of an effective (scalar field dependent) cosmological constant in this

equation. Comparing this result to (4.9), we observe that the effect of a gauging of the scaling symmetry $\mathbb{R}^{+}$is a positive contribution to this effective cosmological constant. The same effect will occur in the corresponding higher-dimensional theories. In standard gravity theories the scalar dependent function $W(\phi)$ would correspond to the scalar potential from which in particular also the scalar masses are derived. This is different in the presence of an $\mathbb{R}^{+}$-gauging: as the resulting theory does in general not admit an action, it is not clear if the mass contributions to the scalar field equations descend from a scalar potential - and we will see in equation (4.44) below explicitly that this is not the case. To this end, we note that the variation of (4.42) is given by

$$
\delta W(\phi)=-\frac{1}{4} g^{2} \Gamma_{A \dot{A}}^{I}\left(3 A_{1}^{I J} A_{2}^{J \dot{A}}-A_{2}^{I \dot{B}} A_{3}^{\dot{A} \dot{B}}+\frac{1}{8} \Gamma_{B \dot{A}}^{J} B_{I J} B_{B}\right) \delta \Sigma^{A},
$$

as can be derived from the differential relations (4.12) upon replacing $\mathcal{P}_{\mu}^{A}$ by $\delta \Sigma^{A}$.

By calculating the supersymmetry variation of the gravitino field equation we have thus fixed all unknown coefficients in this equation and obtained the modified Einstein equation up to its fermionic contributions. The latter may in principle be obtained by repeating the calculation including all higher order fermionic terms. The remaining set of equations of motion are the Dirac equation for the spin-1/2 fields and the scalar field equation. These may be determined in complete analogy to the calculation presented. Rather than going once more through the technical details, we just present the resulting equations:

$$
\begin{aligned}
\gamma^{\mu} \mathcal{D}_{\mu} \chi^{\dot{A}} & =\frac{i}{2} \gamma^{\mu} \gamma^{\nu} \psi_{\mu}^{I} \Gamma_{A \dot{A}}^{I} \mathcal{P}_{\nu}^{A}+g\left(A_{2}^{I \dot{A}}+\frac{1}{4} \Gamma_{A \dot{A}}^{I} B_{A}\right) \gamma^{\mu} \psi_{\mu}^{I}-i g\left(A_{3}^{\dot{A} \dot{B}}+\frac{1}{4} B_{I J} \Gamma_{\dot{A} \dot{B}}^{I J}\right) \chi^{\dot{B}} \\
\mathcal{D}^{\mu} \mathcal{P}_{\mu}^{A} & =\frac{1}{8} g^{2} \Gamma_{A \dot{A}}^{I}\left(3 A_{1}^{I J} A_{2}^{J \dot{A}}-A_{3}^{\dot{A} \dot{B}} A_{2}^{I \dot{B}}+2 B_{I J} A_{2}^{J \dot{A}}-2 \Gamma_{B \dot{A}}^{J} B_{I J} B_{B}\right)
\end{aligned}
$$

The quadratic constraint (4.22) crucially enters in the derivation of these equations. We have thus obtained the full set of deformed equations of motion for the general gauged maximal theory in three dimensions to lowest order in the fermions. Comparing (4.44) to (4.43) one observes that the scalar mass terms (the r.h.s. of (4.44)) for non-vanishing $B_{A}$ do not descend from the potential $W(\phi)$. This is another manifestation of the fact that the resulting theory does not admit an action.

\section{Conclusions and Outlook}

In this paper we have constructed the gaugings of maximal supergravity in which the trombone symmetry (1.1) becomes part of the local gauge symmetries. We have set up the algebraic formalism to describe these theories as an extension of the standard gaugings. More precisely, the gaugings are parametrized by a constant embedding tensor $\widehat{\Theta}_{M}{ }^{\hat{\alpha}}$ which has irreducible components $\left(\Theta_{M}{ }^{\alpha}, \theta_{M}\right)$. In case the second component is zero, $\theta_{M}=0$, these theories reduce to the standard gaugings with gauge group inside 
the duality group G. Non-vanishing $\theta_{M}$ on the other hand amounts to the inclusion of the scaling symmetry (1.1) into the gauge group.

The explicit form of the gauge group generators is given in (2.18) where the value of $\zeta$ has been determined in section 3 for the maximal supergravities in various dimensions. As a result we find that gauging of the scaling symmetry (1.1) necessitates simultaneous gauging of certain generators within the duality group G. We have worked out the algebraic consistency constraints bilinear in the components $\left(\Theta_{M}{ }^{\alpha}, \theta_{M}\right)$. For the particular class of theories with $\Theta_{M}{ }^{\alpha}=0$ (which thus correspond to a "minimal" gauging of the trombone symmetry), we have explicitly constructed the general solution to these consistency constraints. Interestingly, this solution relies on a generalization of the "pure spinor" structure of $\mathrm{SO}(10)$ to the higher-rank exceptional groups.

Finally, we have for the example of the three-dimensional theory worked out the deformed supersymmetry algebra and the full set of equations of motion. In particular, we have shown that gaugings involving the trombone symmetry are compatible with supersymmetry provided the components of the embedding tensor satisfy the aforementioned algebraic consistency constraints. Since these theories in general do no longer admit an action they must be constructed on the level of the equations of motion which are uniquely determined by supersymmetry.

As a generic feature of a gauging of the trombone symmetry we have found a positive contribution to the cosmological constant. The same shows up in the corresponding higher-dimensional theories. The existence of a ten-dimensional de Sitter vacuum in the theory of [13, 14] has been further investigated in [17]. From this point of view it will be interesting to analyze the general structure of the equations of motion and their solutions for the theories with "minimal" gauging of the trombone symmetry given in this paper. Another interesting question is about the structure of theories for which both components $\Theta_{M}{ }^{\alpha}$ and $\theta_{M}$ are non-vanishing. The presence of additional deformation parameters $\theta_{M}$ as compared to the standard gaugings (which moreover give rise to positive contributions in the cosmological constant) may prove useful in the search for stable de Sitter vacua in $N>1$ supersymmetric theories which to date seem extremely rare [37, 38]. Of course, a higher-dimensional interpretation for these additional deformation parameters would be highly desirable.

Let us finally discuss another intriguing aspect about the theories we have constructed. It is well known that the representation in which the embedding tensor transforms under $\mathrm{G}$ in the standard gaugings (column ' $\Theta$ ' of table 1) is the representation dual to the totally antisymmetric $(D-1)$-forms of the theory as predicted from the underlying very extended Kac-Moody algebra $\mathrm{E}_{11}$ [39, 40]. More precisely, the embedding tensor can be identified with the integration constants which arise upon solving the non-dynamical field equations for the $(D-1)$-forms [31]. In contrast, the additional gaugings we have constructed allow for additional components $\theta_{M}$ of the embedding tensor transforming in the representation dual to the vector fields. For these constants there is no dual $(D-1)$-form in the field content of the theories, i.e. an $\mathrm{E}_{11}$ origin of these theories is a priori unclear. However, following the discussion in 
the introduction, the trombone symmetries in the various dimensions seem intimately linked to the duality groups $\mathrm{G}$, such that one would expect that all these gaugings can be cast into a common framework. Indeed, some observations hint in this direction: inspecting a little closer the full field content as predicted by $\mathrm{E}_{11}$, as given in the tables of [40], one observes that there does exist an object in the correct $\mathrm{G}$ representation with $D-1$ space-time indices which however is not an antisymmetric form but a tensor with mixed symmetry $C_{\mu,\left[\nu_{1} \nu_{2} \ldots, \nu_{D-2}\right]}^{M}$. Like the $(D-1)$-forms, such a field does not possess propagating degrees of freedom (see e.g. [41]) and can consistently be set to zero. It is a highly intriguing question if the presence of such tensors with mixed symmetry could in some way trigger the deformations of the presented type.

In fact, the pattern continues: the antisymmetric $D$-forms in standard gaugings turn out to transform under $G$ in the representation which is dual to the quadratic constraint on the embedding tensor [31. As we have shown in this paper, the presence of the additional components $\theta_{M}$ gives rise to additional quadratic constraints, cf. equations (3.13), (3.26), (3.38), (3.52), for the various dimensions. Comparing these additional representations to the tables of [40] we find again a matching of representations with tensors carrying $D$ space-time indices with mixed symmetry structure!

After reduction to $D=2$ dimensions, all these tensors embed into representations of the affine symmetry algebra $E_{9(9)}$. Table 1 shows that remarkably under this algebra there is no longer a difference between the theories triggered by the new parameters $\theta$ and the standard gaugings: both $\Theta$ and $\theta$ combine into a single irreducible (infinite-dimensional) representation of $\mathrm{E}_{9(9)}$ [25], suggesting that also under the bigger algebras $\mathrm{E}_{10}$ and $\mathrm{E}_{11}$ there should be a uniform and common structure underlying all the gaugings.

Along these lines, let us recall that as we have seen throughout the construction, gaugings that involve a local trombone symmetry do no longer admit an action and have thus been constructed on the level of the equations of motion. This is by no means surprising since they involve the gauging of a symmetry that was not off-shell realized. However, a similar fate applies to part of the duality groups $G$ in even spacetime dimensions. E.g. in $D=4$ dimensions (depending on the electric frame chosen) only an $\mathrm{SL}(8)$ subgroup of $\mathrm{G}=\mathrm{E}_{7(7)}$ is realized as a symmetry of the action while the full $E_{7(7)}$ can only be realized on the combined set of equations of motion and Bianchi identities [2]. Nevertheless, in this theory it is possible to gauge subgroups within the full $\mathrm{E}_{7(7)}$ on the level of the action - upon introducing further higher-rank $p$-forms [42]. The same pattern extends to all even dimensions [24, 25, 26]. It would be very exciting (and further complete the presumed $\mathrm{E}_{11}$ picture underlying the theory) if also the theories presented in this paper could be lifted to an action precisely by introducing the additional higher-rank tensors of mixed symmetry mentioned above. In this respect we mention the recent construction of a parent action for the dual graviton - the simplest of all tensors with mixed symmetry — which is based on Stückelberg-type couplings to higher-rank tensor fields in a way reminiscent of the structures appearing in gauged supergravity [43. 


\section{Acknowledgements:}

This work is supported in part by the Agence Nationale de la Recherche (ANR).

\section{Appendix}

\section{A Algebra conventions}

\section{A.1 $\mathrm{E}_{8(8)}$ conventions.}

The algebra $\mathfrak{e}_{8(8)}$ is generated by 248 generators $t_{\mathcal{M}}$

$$
\left[t_{\mathcal{M}}, t_{\mathcal{N}}\right]=f_{\mathcal{M N}}{ }^{\mathcal{K}} t_{\mathcal{K}}
$$

which may be split into 120 compact ones $X^{I J}=-X^{J I}$, corresponding to the maximal compact subalgebra $\mathfrak{s o}(16)$ of the algebra, and 128 non-compact ones $Y^{A}$, with $\mathrm{SO}(16)$ vector indices $I, J, \ldots=1, \ldots, 16$, and spinor indices $A, B, \ldots=1, \ldots, 128$. Dotted indices $\dot{A}, \dot{B}, \ldots$ label the conjugate $\mathrm{SO}(16)$ spinor representation. An extra factor of $\frac{1}{2}$ always appears when summing over antisymmetrized index pairs $[I J]$. $\mathrm{E}_{8(8)}$ indices are raised and lowered by means of the Cartan-Killing metric

$$
\eta_{\mathcal{M N}}=\frac{1}{60} \operatorname{Tr} t_{\mathcal{M}} t_{\mathcal{N}}
$$

In the $\mathrm{SO}(16)$ basis, the components of the Cartan-Killing form are $\eta^{A B}=\delta^{A B}$ and $\eta^{I J, K L}=-2 \delta_{K L}^{I J}$ and the completely antisymmetric structure constants of the algebra are given by

$$
f^{I J, K L, M N}=-8 \delta^{[I[K} \delta_{M N}^{L] J]}, \quad f^{I J, A, B}=-\frac{1}{2} \Gamma_{A B}^{I J}
$$

An important object is the group-valued scalar matrix $\mathcal{V}^{\mathcal{M}} \underline{\mathcal{M}}=\left\{\mathcal{V}^{\mathcal{M}}{ }_{I J}, \mathcal{V}^{\mathcal{M}}{ }_{A}\right\}$. It satisfies

$$
\eta_{\mathcal{M N}} \mathcal{V}^{\mathcal{M}}{ }_{I J} \mathcal{V}^{\mathcal{N}}{ }_{K L}=-2 \delta_{K L}^{I J}, \quad \eta_{\mathcal{M N}} \mathcal{V}^{\mathcal{M}}{ }_{A} \mathcal{V}^{\mathcal{N}}{ }_{B}=\delta_{A B}
$$

which allows to express its inverse explicitly as

$$
\mathcal{V} \underline{\mathcal{M}}_{\mathcal{M}}=\left\{\begin{aligned}
\mathcal{V}^{I J}{ }_{\mathcal{M}} & =-\mathcal{V}_{\mathcal{M}}{ }^{I J} \equiv \eta_{\mathcal{M N}} \mathcal{V}^{\mathcal{N} I J} \\
\mathcal{V}^{A}{ }_{\mathcal{M}} & =\mathcal{V}_{\mathcal{M}}{ }^{A} \equiv \eta_{\mathcal{M N}} \mathcal{V}^{\mathcal{N} A}
\end{aligned}\right.
$$

The fact that the structure constants (A.3) are $\mathrm{E}_{8(8)}$ invariant tensors and thus invariant under contraction with $\mathcal{V}^{\mathcal{M}} \underline{\mathcal{M}}$ is reflected by equations (4.3). 


\section{A.2 $\quad \mathrm{E}_{7(7)}$ conventions and identities.}

The algebra $\mathfrak{e}_{7(7)}$ is generated by 133 generators $t_{\alpha}$

$$
\left[t_{\alpha}, t_{\beta}\right]=f_{\alpha \beta}^{\gamma} t_{\gamma}
$$

Its fundamental representation has dimension 56 ; indices $m, n=1, \ldots, 56$, can be raised and lowered with the symplectic matrix $\Omega_{m n}$ where we use north-west southeast conventions

$$
X^{m}=\Omega^{m n} X_{n}, \quad X_{m}=X^{n} \Omega_{n m}
$$

We raise and lower the adjoint indices $\alpha, \beta=1, \ldots 133$, with the invariant metric $\kappa^{\alpha \beta}=\operatorname{Tr}\left(t^{\alpha} t^{\beta}\right)$ proportional to the Cartan-Killing form. It is related to the structure constants $f_{\alpha \beta}^{\gamma}$ as

$$
f_{\alpha \gamma}{ }^{\delta} f_{\beta \delta}^{\gamma}=3 \kappa_{\gamma \delta}
$$

By performing various contractions, one can prove the non-trivial relation (3.31) between $E_{7(7)}$ generators

$$
\left(t^{\alpha}\right)_{m}{ }^{k}\left(t_{\alpha}\right)_{n}{ }^{l}=\frac{1}{24} \delta_{m}^{k} \delta_{n}^{l}+\frac{1}{12} \delta_{m}^{l} \delta_{n}^{k}+\left(t^{\alpha}\right)_{m n}\left(t_{\alpha}\right)^{k l}-\frac{1}{24} \Omega_{m n} \Omega^{k l} .
$$

E.g., contracting the indices $k$ and $n$, we find in particular

$$
8\left(t^{\alpha}\right)_{m}^{k}\left(t_{\alpha}\right)_{k}^{l}=19 \delta_{m}^{l}
$$

We will need some more identities for this algebra. The first one takes the form

$$
9\left(t^{\alpha}\right)_{m}{ }^{k}\left(t^{\beta}\right)_{k n}\left(t_{\alpha}\right)^{(p q}\left(t_{\beta}\right)^{r s)}+2\left(t^{\alpha}\right)_{[m}{ }^{(r}\left(t_{\alpha}\right)^{p q} \delta_{n]}^{s)}=\frac{1}{8} \Omega_{m n}\left(t^{\alpha}\right)^{(p q}\left(t_{\alpha}\right)^{r s)} .
$$

Note that this identity is antisymmetric in $[\mathrm{mn}]$ and totally symmetric in (pqrs). The existence of such a relation thus follows from the fact that there are only two independent invariant tensors with this index structure (only two singlets in the correspondingly symmetrized tensor product of fundamental representations). The coefficients can be determined by performing various contractions.

In a similar way we obtain another important relation which is totally symmetric in indices $(k \operatorname{lmn} p q)$ :

$$
\left(t^{\beta}\right)^{(k l}\left(t_{\beta}\right)^{m n}\left(t_{\alpha}\right)^{p q)}+8\left(t_{\alpha}\right)^{r s}\left(t^{\beta}\right)_{r}{ }^{(k}\left(t^{\gamma}\right)_{s}^{l}\left(t_{\beta}\right)^{m n}\left(t_{\gamma}\right)^{p q)}=0
$$

\section{A.3 $\mathrm{E}_{8(8)}$ algebra in the $\mathrm{E}_{7(7)} \times \mathrm{SL}(2)$ basis}

Under its maximal subgroup $\mathrm{E}_{7(7)} \times \mathrm{SL}(2)$, the adjoint representation of $\mathrm{E}_{8(8)}$ breaks as

$$
248 \longrightarrow(133,1) \oplus(56,2) \oplus(1,3)
$$


Accordingly, we split the generators $t_{\mathcal{M}}$ into $t_{\alpha}, t_{m, a}$ and $t_{(a b)}$, where $m$ and $\alpha$ denote the fundamental and the adjoint representation of $\mathrm{E}_{7(7)}$, respectively, while $a, b$ denote the doublet of SL(2). In these generators, the algebra takes the form

$$
\begin{aligned}
{\left[t_{\alpha}, t_{\beta}\right] } & =f_{\alpha \beta} \delta t_{\delta}, \quad\left[t_{(a b)}, t_{(c d)}\right]=2 \delta_{(a}^{e} \epsilon_{b)(c} \delta_{d)}^{f} t_{(e f)}, \\
{\left[t_{m, a}, t_{(b c)}\right] } & =\epsilon_{a(b} t_{m, c)}, \quad\left[t_{m, a}, t_{\alpha}\right]=\left(t_{\alpha}\right)_{m}^{n} t_{n, a}, \\
{\left[t_{m, a}, t_{n, b}\right] } & =\frac{1}{12} \Omega_{m n} t_{(a b)}+\epsilon_{a b}\left(t^{\alpha}\right)_{m n} t_{\alpha} .
\end{aligned}
$$

Here, we use the $\mathrm{E}_{7(7)}$-invariant tensors introduced in section $\mathrm{A} .2$ and the $\mathrm{SL}(2)$ invariant $\epsilon$-symbol $\epsilon^{a b}$.

\section{B Solution of the $\mathrm{E}_{8(8)}$ constraint (3.53)}

A particular class of gaugings we have studied in this paper are those theories which are triggered by a single constant vector $\theta_{M}$. In three dimensions, this vector is subject to the quadratic constraint (3.53)

$$
\left(\left(\mathbb{P}_{\mathbf{1}}\right)_{\mathcal{M N}}{ }^{\mathcal{K L}}+\left(\mathbb{P}_{\mathbf{3 8 7 5}}\right)_{\mathcal{M N}}{ }^{\mathcal{K} \mathcal{L}}\right) \theta_{\mathcal{K}} \theta_{\mathcal{L}} \stackrel{!}{\equiv} 0
$$

In this appendix we will analyze in detail this quadratic constraint and derive its general solution given in (3.55) in the main text. Explicitly, the constraint (B.1) reads

$$
\theta^{\mathcal{M}} \theta_{\mathcal{M}}=0, \quad \theta_{\mathcal{M}} \theta_{\mathcal{N}}-\frac{1}{2} f^{\mathcal{Q P}}{ }_{\mathcal{M}} f_{\mathcal{N} \mathcal{Q}}{ }^{\mathcal{L}} \theta_{\mathcal{P}} \theta_{\mathcal{L}}=0
$$

\section{B.1 The constraint under $\mathrm{E}_{7(7)} \times \mathrm{SL}(2)$}

In order to solve the constraint (B.1), we employ the same technique that allowed to explicitly solve the pure spinor constraint (3.14) in $D=6$ and its analogues (3.27), (3.39) in $D=5$ and $D=4$, respectively. It is useful to first break $\mathrm{E}_{8(8)}$ under its subgroup $\mathrm{E}_{7(7)} \times \mathrm{SL}(2)$ as given explicitly in section A.3 above. The adjoint representation breaks according to (A.13) such that we can parametrize the vector $\theta_{\mathcal{M}}$ by components $\left\{\theta_{\alpha}, \theta_{m, a}, \theta_{(a b)}\right\}$. The constraint (B.1) under this subgroup breaks into

$$
1 \oplus 3875 \longrightarrow 2 \cdot(\mathbf{1}, \mathbf{1}) \oplus(\mathbf{1 5 3 9}, \mathbf{1}) \oplus(56,2) \oplus(\mathbf{9 1 2}, \mathbf{2}) \oplus(\mathbf{1 3 3}, 3) .
$$

As a first step we will express this constraint explicitly in terms of the $\mathrm{E}_{7(7)} \times \mathrm{SL}(2)$ components $\left\{\theta_{\alpha}, \theta_{m, a}, \theta_{(a b)}\right\}$. To this end, we start from the general $\mathrm{E}_{7(7)} \times \mathrm{SL}(2)$ singlet bilinear in the components of $\theta_{\mathcal{M}}$

$$
\Phi_{\sigma, \tau} \equiv \theta^{(c d)} \theta_{(c d)}+\sigma \epsilon^{c d} \Omega^{k p} \theta_{k, c} \theta_{p, d}+\tau \theta_{\alpha} \theta^{\alpha}
$$

labeled by two relative coefficients $\sigma, \tau$. With respect to $\mathrm{E}_{8(8)}$, the general bilinear expression in $\theta_{\mathcal{M}}$ transforms in the representation

$$
(248 \otimes 248)_{\mathrm{sym}}=1 \oplus 3875 \oplus 27000 .
$$


In order to identify the constraint (B.1) we seek within the three linearly independent singlets (B.4) the two combinations corresponding to the r.h.s. of (B.3), i.e. the two singlets descending from the $\mathrm{E}_{8(8)}$ representations $\mathbf{1}$ and $\mathbf{3 8 7 5}$ - while the third combination corresponds to the singlet descending from the $\mathbf{2 7 0 0 0 .}$

To this end we compute the action of an $\mathrm{E}_{8(8)}$ generator $t_{m, a}$ on $\Phi_{\sigma, \tau}$. As $\theta_{\mathcal{M}}$ transforms in the adjoint representation of $\mathrm{E}_{8(8)}$, this action can directly be deduced from (A.14). The result is given by

$$
t_{m, a} \cdot \Phi_{\sigma, \tau}=\frac{1}{6}(\sigma-12) \epsilon^{c d} \theta_{m, c} \theta_{(a d)}+2(\sigma+\tau)\left(t^{\alpha}\right)_{m}{ }^{n} \theta_{n, a} \theta_{\alpha} .
$$

This shows that $\Phi_{12,-12}$ is an $\mathrm{E}_{8(8)}$ singlet, i.e. we have identified the singlet descending from the 1 of $\mathrm{E}_{8(8)}$. Applying another generator $t_{n, b}$ on (B.6) and contracting all free indices gives rise to

$$
\begin{aligned}
\Omega^{n m} \epsilon^{a b} t_{n, b} \cdot t_{m, a} \cdot \Phi_{\sigma, \tau}= & \frac{7}{9}(12-\sigma) \theta^{(a b)} \theta_{(a b)}+4(\sigma+\tau) \theta^{\alpha} \theta_{\alpha} \\
& -\frac{1}{4}(12-20 \sigma-19 \tau) \Omega^{m n} \epsilon^{a b} \theta_{m, a} \theta_{n, b} .
\end{aligned}
$$

Note that the operator $\mathcal{C} \equiv \Omega^{n m} \epsilon^{a b} t_{n, b} t_{m, a}$ acting on $\Phi_{\sigma, \tau}$ is proportional to the quadratic Casimir of $\mathrm{E}_{8(8)}$. Diagonalizing its action (B.7) we find

$$
\mathcal{C} \cdot \Phi_{12,-12}=0, \quad \mathcal{C} \cdot \Phi_{\frac{12}{7}, \frac{12}{7}}=8 \Phi_{\frac{12}{7}, \frac{12}{7}}, \quad \mathcal{C} \cdot \Phi_{-\frac{9}{7},-\frac{108}{133}}=\frac{31}{3} \Phi_{-\frac{9}{7},-\frac{108}{133}},
$$

and can thereby identify the singlets $\Phi_{12,-12}$ and $\Phi_{\frac{12}{7}}, \frac{12}{7}$ descending from the $\mathbf{1}$ and the 3875 of $\mathrm{E}_{8(8)}$, respectively.

The full constraint (B.1) can thus be obtained as the $\mathrm{E}_{8(8)}$ orbit of the second singlet. The action of two $\mathrm{E}_{8(8)}$ generators on $\Phi_{\frac{12}{7}}, \frac{12}{7}$ is given by

$$
\begin{aligned}
7 t_{n, b} \cdot t_{m, a} \cdot \Phi_{\frac{12}{7}, \frac{12}{7}}= & -\Omega_{n m} \epsilon^{c d} \theta_{(b c)} \theta_{(a d)}+\left(t^{\alpha}\right)_{n m} \theta_{\alpha} \theta_{(a b)} \\
& -6 \epsilon_{b a} \epsilon^{c d} \theta_{m, c} \theta_{n, d}-6 \theta_{m, b} \theta_{n, a} \\
& +48 \epsilon_{b a}\left(t^{\alpha}\right)_{m}{ }^{k}\left(t_{\beta}\right)_{k n} \theta_{\beta} \theta_{\alpha}+48\left(t^{\alpha}\right)_{m}{ }^{k}\left(t_{\beta}\right)_{n}{ }^{p} \theta_{k, a} \theta_{p, b} .
\end{aligned}
$$

By various contractions one finds from this equation and from (B.6) the different parts of (B.3). As a result, we give the constraint (B.1) explicitly in terms of the components $\left\{\theta_{\alpha}, \theta_{m, a}, \theta_{(a b)}\right\}$ :

$$
\begin{array}{rlll}
\theta^{(c d)} \theta_{(c d)}+12 \epsilon^{c d} \Omega^{k p} \theta_{k, c} \theta_{p, d}-12 \theta_{\alpha} \theta^{\alpha} & \stackrel{!}{\equiv} 0, & (\mathbf{1}, \mathbf{1})_{(1)} \\
7 \theta^{(c d)} \theta_{(c d)}+12 \epsilon^{c d} \Omega^{k p} \theta_{k, c} \theta_{p, d}+12 \theta_{\alpha} \theta^{\alpha} & \stackrel{!}{\equiv} 0, & (\mathbf{1}, \mathbf{1})_{(3875)} \\
\epsilon^{c d} \theta_{m, c} \theta_{(a d)}-4\left(t^{\alpha}\right)_{m}{ }^{n} \theta_{n, a} \theta_{\alpha} & \stackrel{!}{\equiv} 0, & (\mathbf{5 6}, \mathbf{2}) \\
\theta_{\alpha} \theta_{(a b)}+6\left(t_{\alpha}\right)^{m n} \theta_{m, a} \theta_{n, b} & \stackrel{!}{\equiv} 0, & (\mathbf{1 3 3}, \mathbf{3}) \\
\epsilon^{a b} \theta_{[m, a} \theta_{n], b}-6\left(t^{\alpha}\right)_{[m}{ }^{k}\left(t^{\beta}\right)_{n] k} \theta_{\alpha} \theta_{\beta}-\text { trace } \stackrel{!}{\equiv} 0 & (\mathbf{1 5 3 9 , 1 )}
\end{array}
$$

We have left out the constraint in the $(\mathbf{9 1 2}, \mathbf{2})$ which is obtained by the action of three $\mathrm{E}_{8(8)}$ generators on $\Phi_{\frac{12}{7}, \frac{12}{7}}$. As we shall see in the next section, this part of the constraint is automatically satisfied and does not lead to new constraints. 


\section{B.2 Solving the constraint}

For the explicit solution of $(\underline{B .10})$, we further break these equations under $\mathrm{E}_{7(7)} \times$ $\mathbb{R}^{+}$. According to the decomposition (3.54), we break the vector $\theta_{\mathcal{M}}$ into components $\left(\tilde{\eta}, \tilde{\eta}_{m}, \xi, \xi_{\alpha}, \eta_{m}, \eta\right)$ defined as

$$
\begin{aligned}
\tilde{\eta} & \equiv \theta_{(++)}, \quad \xi \equiv \theta_{(+-)}, \quad \eta \equiv \theta_{(--)} \\
\tilde{\eta}_{m} & \equiv \theta_{m,+}, \quad \eta_{m} \equiv \theta_{m,-}, \quad \xi_{\alpha} \equiv \theta_{\alpha},
\end{aligned}
$$

where we have broken up the $\mathrm{SL}(2)$ components introduced in the last subsection. In terms of these components, the full set of constraints (B.10) takes the form

$$
\begin{aligned}
& \eta \tilde{\eta}-\xi^{2}+12 \Omega^{m n} \tilde{\eta}_{m} \eta_{n}-6 \xi_{\alpha} \xi^{\alpha} \stackrel{!}{\equiv} 0, \quad\left(\mathbf{1}_{(1)}^{0}\right) \\
& 7 \eta \tilde{\eta}-7 \xi^{2}+12 \Omega^{m n} \tilde{\eta}_{m} \eta_{n}+6 \xi_{\alpha} \xi^{\alpha} \stackrel{!}{\equiv} 0, \quad\left(\mathbf{1}_{(3875)}^{0}\right) \\
& \tilde{\eta} \eta_{m}-\xi \tilde{\eta}_{m}+4\left(t^{\alpha}\right)_{m}{ }^{n} \xi_{\alpha} \tilde{\eta}_{n} \stackrel{!}{\equiv} 0, \quad\left(56^{+1}\right) \\
& \eta \tilde{\eta}_{m}-\xi \eta_{m}-4\left(t^{\alpha}\right)_{m}^{n} \xi_{\alpha} \eta_{n} \stackrel{!}{\equiv} 0, \quad\left(56^{-1}\right) \\
& \xi_{\alpha} \tilde{\eta}+6\left(t_{\alpha}\right)^{m n} \tilde{\eta}_{m} \tilde{\eta}_{n} \stackrel{!}{\equiv} 0, \quad\left(133^{+2}\right) \\
& \xi_{\alpha} \xi+6\left(t_{\alpha}\right)^{m n} \tilde{\eta}_{m} \eta_{n} \stackrel{!}{\equiv} 0, \quad\left(133^{0}\right) \\
& \xi_{\alpha} \eta+6\left(t_{\alpha}\right)^{m n} \eta_{m} \eta_{n} \stackrel{!}{\equiv} 0, \quad\left(133^{-2}\right) \\
& \tilde{\eta}_{[m} \eta_{n]}-\frac{1}{56} \Omega_{m n} \Omega^{k p} \tilde{\eta}_{k} \eta_{p}-3\left(t^{\alpha}\right)_{[m}{ }^{k}\left(t^{\beta}\right)_{n] k} \xi_{\beta} \xi_{\alpha}+\frac{3}{56} \Omega_{m n} \xi^{\alpha} \xi_{\alpha} \stackrel{!}{\equiv} 0, \quad\left(\mathbf{1 5 3 9} 9^{0}\right)
\end{aligned}
$$

where again we have left out the two equations in the $\mathbf{9 1 2}^{ \pm 1}$ which we will justify shortly.

In analogy to the higher-dimensional cases, we start from a given set of 57 parameters $\eta, \eta_{m}$ and try to determine the remaining ones by virtue of (B.12). Equation $\left(133^{-2}\right)$ directly determines $\xi_{\alpha}$

$$
\xi_{\alpha}=-\frac{6}{\eta}\left(t_{\alpha}\right)^{m n} \eta_{m} \eta_{n}
$$

With $\left(56^{-1}\right)$, we find for $\tilde{\eta}_{m}$

$$
\tilde{\eta}_{m}=\frac{\xi}{\eta} \eta_{m}-\frac{24}{\eta^{2}}\left(t^{\alpha}\right)_{m}^{n}\left(t_{\alpha}\right)^{p q} \eta_{n} \eta_{p} \eta_{q}
$$

Equation $\left(\mathbf{1 3 3}^{0}\right)$ is then automatically satisfied. Its verification requires the vanishing of the term quartic in $\eta_{m}$ which simply follows from the absence of an adjoint representation in the totally symmetric tensor product $\left(\mathbf{5 6} \mathbf{6}^{\otimes 4}\right)_{\mathrm{sym}}$.

Continuing with the $\mathbf{1 3 3}^{\mathbf{+ 2}}$, we obtain after using (B.13), (B.14)

$$
\tilde{\eta} \xi_{\alpha}=-\frac{6}{\eta^{2}}\left(t_{\alpha}\right)^{m n} \eta_{m} \eta_{n} \xi^{2}-\frac{96}{\eta^{2}}\left(t_{\alpha}\right)^{m n}\left(t^{\beta}\right)_{m}{ }^{k}\left(t^{\gamma}\right)_{n}{ }^{l} \eta_{k} \eta_{l} \xi_{\beta} \xi_{\gamma}
$$


The last term can be simplified by means of the identity (A.12). Multiplying the latter with six $\eta_{m}$ 's, we find

$$
\left(t_{\alpha}\right)^{m n}\left(t^{\beta}\right)_{m}{ }^{k}\left(t^{\gamma}\right)_{n}{ }^{l} \eta_{k} \eta_{l} \xi_{\beta} \xi_{\gamma}=\frac{\eta}{48} \xi_{\beta} \xi^{\beta} \xi_{\alpha}
$$

Equation (B.15) can be solved by setting

$$
\tilde{\eta}=\frac{\xi^{2}}{\eta}-\frac{2}{\eta} \xi_{\alpha} \xi^{\alpha} .
$$

We have thus determined all unknown parameters and verified the solution (3.55). It is straightforward to check, that the two singlets in (B.12) are automatically satisfied with (B.13), (B.14), (B.16). Furthermore, the $\mathbf{5 6} \mathbf{6}^{+\mathbf{1}}$ reduces to

$$
0=\frac{2}{\eta}\left(\eta_{m} \xi^{\beta} \xi_{\beta}-8\left(t^{\alpha}\right)_{m}^{n}\left(t^{\beta}\right)_{n}^{k} \eta_{k} \xi_{\alpha} \xi_{\beta}\right)
$$

which can be verified upon multiplying the identity (A.11) with five $\eta_{m}$ 's. Finally, the $1539^{0}$ reduces to

$$
\begin{aligned}
0=- & \frac{12}{\eta^{2}}\left(2 \eta_{[n}\left(t^{\alpha}\right)_{m]}^{p}\left(t_{\alpha}\right)^{q r} \eta_{p} \eta_{q} \eta_{r}+9\left(t^{\alpha}\right)_{m}^{k}\left(t^{\beta}\right)_{n k}\left(t_{\beta}\right)^{p q}\left(t_{\alpha}\right)^{r s} \eta_{p} \eta_{q} \eta_{r} \eta_{s}\right) \\
& - \text { trace }
\end{aligned}
$$

which is another consequence of (A.11).

We have thus verified, that the solution (B.13), (B.14), (B.16) satisfies all constraint equations (B.12). In principle, there are two more equations to verify which transform in the $\mathbf{9 1 2}^{ \pm 1}$. However, with the given solution all constraint equations translate into relations among a product of $\eta_{m}$ 's transforming in the fundamental 56. Since there is no 912 representation in the corresponding completely symmetrized tensor products of the fundamental 56, every such constraint is automatically satisfied. This finishes the proof of (3.55).

\section{References}

[1] E. Cremmer, H. Lu, C. N. Pope, and K. S. Stelle, Spectrum-generating symmetries for BPS solitons, Nucl. Phys. B520 (1998) 132-156, hep-th/9707207.

[2] E. Cremmer and B. Julia, The SO(8) supergravity, Nucl. Phys. B159 (1979) 141.

[3] E. Cremmer, B. Julia, H. Lu, and C. N. Pope, Dualisation of dualities. I, Nucl. Phys. B523 (1998) 73-144, hep-th/9710119.

[4] E. Cremmer, B. Julia, and J. Scherk, Supergravity theory in 11 dimensions, Phys. Lett. B76 (1978) 409-412. 
[5] B. de Wit and H. Nicolai, $N=8$ supergravity, Nucl. Phys. B208 (1982) 323.

[6] M. Günaydin, L. J. Romans, and N. P. Warner, Compact and noncompact gauged supergravity theories in five-dimensions, Nucl. Phys. B272 (1986) 598.

[7] M. Pernici, K. Pilch, and P. van Nieuwenhuizen, Gauged maximally extended supergravity in seven-dimensions, Phys. Lett. B143 (1984) 103.

[8] H. Nicolai and H. Samtleben, Maximal gauged supergravity in three dimensions, Phys. Rev. Lett. 86 (2001) 1686-1689, hep-th/0010076); Compact and noncompact gauged maximal supergravities in three dimensions, JHEP 04 (2001) 022, hep-th/0103032.

[9] B. de Wit, H. Samtleben, and M. Trigiante, On Lagrangians and gaugings of maximal supergravities, Nucl. Phys. B655 (2003) 93-126, hep-th/0212239.

[10] B. de Wit, H. Samtleben, and M. Trigiante, The maximal $D=5$ supergravities, Nucl. Phys. B716 (2005) 215-247, hep-th/0412173.

[11] B. de Wit and H. Samtleben, Gauged maximal supergravities and hierarchies of nonabelian vector-tensor systems, Fortschr. Phys. 53 (2005) 442-449, hep-th/0501243.

[12] J. Scherk and J. H. Schwarz, How to get masses from extra dimensions, Nucl. Phys. B153 (1979) 61-88.

[13] P. S. Howe, N. D. Lambert, and P. C. West, A new massive type IIA supergravity from compactification, Phys. Lett. B416 (1998) 303-308, hep-th/9707139.

[14] I. V. Lavrinenko, H. Lu, and C. N. Pope, Fibre bundles and generalised dimensional reductions, Class. Quant. Grav. 15 (1998) 2239-2256, hep-th/9710243.

[15] L. J. Romans, Massive $N=2 a$ supergravity in ten-dimensions, Phys. Lett. B169 (1986) 374.

[16] A. Chamblin, M. J. Perry, and H. S. Reall, Non-BPS D8-branes and dynamic domain walls in massive IIA supergravities, JHEP 09 (1999) 014, hep-th/9908047.

[17] A. Chamblin and N. D. Lambert, de Sitter space from M-theory, Phys. Lett. B508 (2001) 369-374, hep-th/0102159.

[18] A. Chamblin and N. D. Lambert, Zero-branes, quantum mechanics and the cosmological constant, Phys. Rev. D65 (2002) 066002, hep-th/0107031.

[19] J. Gheerardyn, Solutions to the massive HLW IIA supergravity, Phys. Lett. B555 (2003) 264-270, hep-th/0211192.

[20] E. Bergshoeff, T. de Wit, U. Gran, R. Linares, and D. Roest, (Non-)Abelian gauged supergravities in nine dimensions, JHEP 10 (2002) 061, hep-th/0209205. 
[21] J. Kerimo and H. Lu, New $D=6, N=(1,1)$ gauged supergravity with supersymmetric Minkowski $4 \times S^{2}$ vacuum, Phys. Lett. B576 (2003) 219-226, hep-th/0307222.

[22] J. Kerimo, J. T. Liu, H. Lu, and C. N. Pope, Variant $N=(1,1)$ supergravity and (Minkowski) ${ }_{4} \times S^{2}$ vacua, Class. Quant. Grav. 21 (2004) 3287-3300, hep-th/0401001.

[23] H. Samtleben and M. Weidner, The maximal $D=7$ supergravities, Nucl. Phys. B 725 (2005) 383, hep-th/0506237.

[24] B. de Wit, H. Samtleben, and M. Trigiante, The maximal $D=4$ supergravities, JHEP 06 (2007) 049, arXiv:0705.2101 [hep-th].

[25] H. Samtleben and M. Weidner, Gauging hidden symmetries in two dimensions, JHEP 08 (2007) 076, arXiv:0705.2606 [hep-th].

[26] E. Bergshoeff, H. Samtleben, and E. Sezgin, The gaugings of maximal $D=6$ supergravity, JHEP 03 (2008) 068, arXiv:0712.4277 [hep-th]].

[27] B. Julia, Infinite Lie algebras in physics, in Johns Hopkins Workshop on Current Problems in Particle Theory, 1981.

[28] T. Damour, M. Henneaux, and H. Nicolai, $\mathrm{E}_{10}$ and a 'small tension expansion' of M theory, Phys. Rev. Lett. 89 (2002) 221601, hep-th/0207267.

[29] P. C. West, E $E_{11}$ and M theory, Class. Quant. Grav. 18 (2001) 4443-4460, hep-th/0104081.

[30] J. Schön and M. Weidner, Gauged $N=4$ supergravities, JHEP 05 (2006) 034, hep-th/0602024.

[31] B. de Wit, H. Nicolai, and H. Samtleben, Gauged supergravities, tensor hierarchies, and M-theory, JHEP 02 (2008) 044, arXiv:0801.1294 [hep-th]].

[32] Y. Tanii, $N=8$ supergravity in six-dimensions, Phys. Lett. B145 (1984) 197-200.

[33] E. Cremmer, Supergravities in 5 dimensions, in Superspace and supergravity : proceedings (S. Hawking and M. Rocek., eds.), Cambridge Univ. Press, 1980. Nuffield Gravity Workshop, Cambridge.

[34] M. Günaydin, K. Koepsell, and H. Nicolai, Conformal and quasiconformal realizations of exceptional Lie groups, Commun. Math. Phys. 221 (2001) 57-76, hep-th/0008063.

[35] N. Marcus and J. H. Schwarz, Three-dimensional supergravity theories, Nucl. Phys. B228 (1983) 145.

[36] K. Peeters, A field-theory motivated approach to symbolic computer algebra, Comput. Phys. Commun. 176 (2007) 550-558, cs/0608005; Introducing Cadabra: A symbolic computer algebra system for field theory problems, hep-th/0701238. 
[37] P. Fré, M. Trigiante, and A. Van Proeyen, Stable de Sitter vacua from $N=2$ supergravity, Class. Quant. Grav. 19 (2002) 4167-4194, hep-th/0205119.

[38] M. de Roo, D. B. Westra, S. Panda, and M. Trigiante, Potential and mass-matrix in gauged $N=4$ supergravity, JHEP 11 (2003) 022, hep-th/0310187.

[39] F. Riccioni and P. West, The $\mathrm{E}_{11}$ origin of all maximal supergravities, JHEP 07 (2007) 063, arXiv:0705.0752 [hep-th].

[40] E. A. Bergshoeff, I. De Baetselier, and T. A. Nutma, E $E_{11}$ and the embedding tensor, JHEP 09 (2007) 047, arXiv:0705.1304 [hep-th].

[41] X. Bekaert and N. Boulanger, Tensor gauge fields in arbitrary representations of $G L(D, \mathbb{R})$ : duality and Poincaré lemma, Commun. Math. Phys. 245 (2004) 27-67, hep-th/0208058.

[42] B. de Wit, H. Samtleben, and M. Trigiante, Magnetic charges in local field theory, JHEP 09 (2005) 016, hep-th/0507289.

[43] N. Boulanger and O. Hohm, Non-linear parent action and dual gravity, Phys. Rev. D78, (2008) 064027, arXiv:0806.2775 [hep-th].

[44] E. A. Bergshoeff, O. Hohm and T. A. Nutma, A Note on E11 and Three-dimensional Gauged Supergravity, JHEP 05 (2008) 081, arXiv:0803.2989 [hep-th]. 